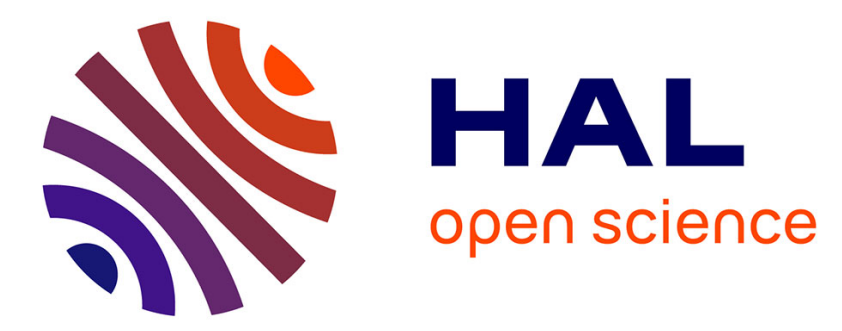

\title{
Nonlinear disturbance evolution in a two-dimensional boundary layer along an elastic plate and induced radiated noise
}

Marie-Laure Gobert, Uwe Ehrenstein, Jacques-André Astolfi, Patrick Bot

\section{- To cite this version:}

Marie-Laure Gobert, Uwe Ehrenstein, Jacques-André Astolfi, Patrick Bot. Nonlinear disturbance evolution in a two-dimensional boundary layer along an elastic plate and induced radiated noise. European Journal of Mechanics - B/Fluids, 2010, 29 (2), pp.105-118. 10.1016/j.euromechflu.2009.12.001 . hal-00433149

\author{
HAL Id: hal-00433149 \\ https://hal.science/hal-00433149
}

Submitted on 18 Nov 2009

HAL is a multi-disciplinary open access archive for the deposit and dissemination of scientific research documents, whether they are published or not. The documents may come from teaching and research institutions in France or abroad, or from public or private research centers.
L'archive ouverte pluridisciplinaire HAL, est destinée au dépôt et à la diffusion de documents scientifiques de niveau recherche, publiés ou non, émanant des établissements d'enseignement et de recherche français ou étrangers, des laboratoires publics ou privés. 


\title{
Nonlinear disturbance evolution in a two-dimensional boundary layer along an elastic plate and induced radiated sound
}

\author{
M.-L. Gobert ${ }^{\mathrm{a}, *}$, U. Ehrenstein ${ }^{\mathrm{b}}$, J. A. Astolfia ${ }^{\mathrm{a}}$, P. Bot $^{\mathrm{a}}$ \\ ${ }^{a}$ IRENav, BCRM Brest, CC 600, F-29240 Brest Cedex 9, France \\ ${ }^{b}$ IRPHÉ UMR 6594, Aix-Marseille Université, CNRS, 49 rue Joliot-Curie, F-13384 \\ Marseille Cedex 13, France
}

\begin{abstract}
The interaction between a boundary-layer flow and an elastic plate is addressed by direct numerical simulation, taking into account the full coupling between the fluid flow and the flexible wall. The convectively unstable flow state is harmonically forced and two-dimensional nonlinearly saturated wavelike disturbances are computed along archetype-plates with respect to stiffness and natural frequencies. In the aim of determining the low-Mach number radiated sound for the system, the simulation data are used to solve the Lighthill's equation in terms of a Green function in the wavenumberfrequency space. Different degrees of fluid-structure coupling are implemented in the radiated sound model and the resulting acoustic pressure levels are compared. The sound radiation levels are shown to be increased in the presence of flexible walls with however significant differences in the radiated pressure levels for different coupling assumptions.
\end{abstract}

\footnotetext{
*Corresponding author. Tel.: +33 2982346 90; Fax: +33 298233857

Email address: marie-laure.gobert@ecole-navale.fr (M.-L. Gobert)
} 
Key words: boundary layer flow over flexible plates, numerical simulation, radiated sound, Lighthill's analogy

\section{Introduction}

An increasing effort is devoted to the understanding, the modeling and the reduction of the self-noise received by sonar antennas and flank arrays, that equip surface ships as well as underwater vehicles. In that context, the hydrodynamic noise, generated by the boundary layer flow that develops along their surface covering, is given a particular attention. The models available are based on wavenumber-frequency spectrum models for the turbulent pressure fluctuation, such as the Corcos' model (cf. Corcos [1]), which has been subsequently improved by Chase [2, 3], and Ffowcs Williams [4], among others. For the archetype configuration of a boundary-layer flow over an elastic plate, it is generally assumed that the pressure field at the fluid-structure interface is the sum of the turbulent pressure, which would be observed for a flow along a rigid plate, and the acoustic pressure induced by the plate motion. The reliability of this weak coupling assumption has been addressed by Graham [5], in the context of aeroacoustics. While the weak-coupling hypothesis appears to be satisfactory for subsonic flows, it is shown in this latter work that there may be discrepancies when considering supersonic flow regimes. More recently, and considering a low Mach-number two-dimensional turbulent boundary layer, Zheng [6, 7], clarified the effect of an elastic plate on radiated sound in the framework of the weak coupling approximation. Applying the celebrated Lighthill's analogy [8, 9], Zheng determined the pressure fluctuation outside the turbulent boundary layer using 
a Green function for appropriate boundary conditions at the compliant wall which has been derived from Dowling [10]. The pressure fluctuation at the outside edge of the boundary layer provides the boundary condition for the homogeneous wave equation, whose solution is the radiated sound in the uniform flow region. It has indeed been shown by Tam [11] that if the Mach number of the mean flow is small, the simple acoustic wave equation can be used, rather than the convected wave equation. Applying the model to the case of an infinite steel plate, Zheng showed that the radiated pressure frequency spectrum over the flexible surface has much higher levels than the rigid one in the low frequency range.

The present work readdresses the sound radiation issue, by considering a strong coupling between the boundary layer and an elastic plate of finite length. Such systems have been extensively studied from a mere stability point of view, through stability analyses as well as spatial numerical simulations. It has been shown that, depending on the plate and flow characteristics, the transition could be either delayed by stabilization of the Tollmien-Schlichting waves interacting with the plate motion, or triggered by surface-induced flow instabilities (see for instance Carpenter \& Garrad [12], Davies \& Carpenter [13], Gad-el Hak [14], Wiplier \& Ehrenstein [15], to cite a few). Here, we compute the two-dimensional flow, coupling the perturbed incompressible Navier-Stokes system with the elastic plate dynamics at a highly supercritical Reynolds number. The computed flow states provide the source terms for the radiated sound analysis in the framework of the Lighthill's analogy. It is hence assumed that in the disturbance region inside the boundary layer, the source terms, computed as incompressible quantities, 
decouple from the acoustic disturbances. This is generally considered as a reliable approximation for low-Mach number flows (cf. Wang, Lele \& Moin [16]). In realistic boundary layers at supercritical Reynolds numbers the flow is three-dimensional and turbulent. However, the direct numerical simulation of a turbulent flow bounded by a compliant wall is still challenging. $\mathrm{Xu}$, Rempfer \& Lumley [17] for instance addressed a turbulent channel flow in the presence of a compliant wall. To limit the computational effort, in the latter work a so-called minimal flow unit with respect to the turbulent flow structure is considered. The aim of the present investigation is to address different coupling assumptions between the flow and a compliant coating in the radiated pressure model. The simplified case of two-dimensional nonlinear disturbances in the fluid-structure system is numerically tractable without any simplifying assumption regarding the fluid-structure coupling. Also, as discussed for instance by Guo [18], two-dimensional analyses may be significant for acoustic problems in engineering application.

The paper is organized as follows. Section 2 is devoted to the description of the numerical tools. In section 3 simulation results for the coupled fluidstructure system are analyzed for two different elastic plates. The pressure fluctuation and Lighthill's tensors are computed and are used in section 4 to solve Lighthill's equation. Radiated sound is computed and comparisons are provided for different coupling assumptions. Some conclusions are drawn in section 5 . 


\section{Numerical tools}

\subsection{Fluid-structure system}

For a supercritical flow regime, the fluid-structure system is solved and the time-integration of the coupled system provides the flow-field used in the subsequent radiated noise analysis. We consider a two-dimensional boundary layer flow that develops along an elastic plate of finite length, clamped into a rigid wall at both ends. The flow is solution of the incompressible NavierStokes equations inside the fluid domain, whose bottom boundary consists of a clamped wall. The simulation domain is therefore defined as $x_{i} \leq x \leq x_{o}$, $\eta(x, t) \leq y \leq \infty$, where $x_{i}$ and $x_{o}$ are respectively the inflow and outflow boundaries of the flow domain in the streamwise $x$ direction, $y$ is the wallnormal coordinate and $\eta$ is the wall displacement (cf. figure 1).

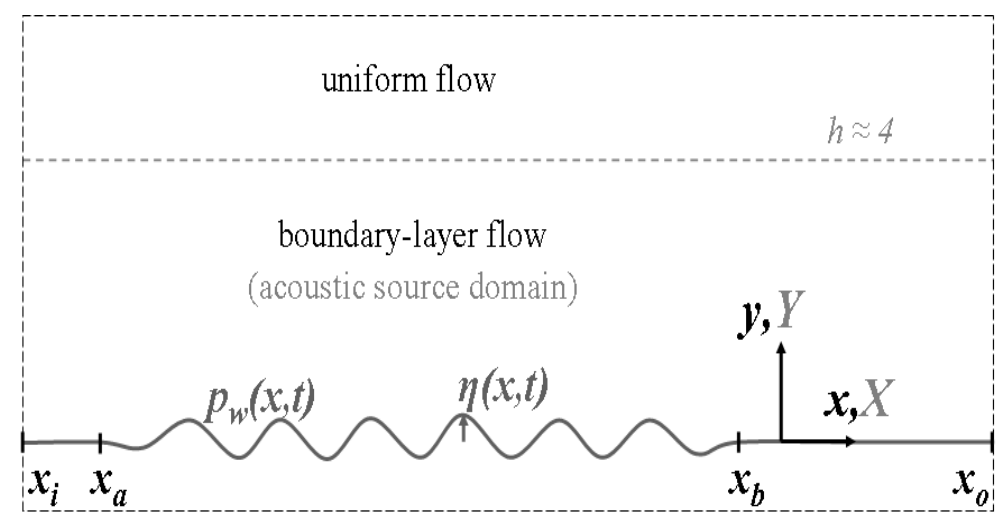

Figure 1: Simulation domain.

The flow domain is transformed into a Cartesian computational domain, using the time-dependent mapping

$$
\bar{x}=x \quad \bar{y}=y-\eta(x, t) \quad \bar{t}=t .
$$


The spatial and time operators of the Navier-Stokes system are then modified by the addition of terms resulting from the mapping and the dimensionless equations are

$$
\begin{aligned}
\frac{\partial \mathbf{u}}{\partial \bar{t}}+(\mathbf{u} \cdot \bar{\nabla}) \mathbf{u}+\bar{\nabla} p-\frac{1}{R e} \bar{\nabla}^{2} \mathbf{u} & =S(\eta, \mathbf{u}, p), \\
\bar{\nabla} \cdot \mathbf{u} & =-\mathbf{G}_{\eta} \cdot \mathbf{u} .
\end{aligned}
$$

In the above system $\bar{\nabla}$ is the Cartesian gradient and $S$ contains all terms depending on $\eta$ with

$$
S(\eta, \mathbf{u}, p)=-T_{\eta} \mathbf{u}-\left(\mathbf{u} \cdot \mathbf{G}_{\eta}\right) \mathbf{u}-\mathbf{G}_{\eta} p+\frac{1}{R e} L_{\eta} \mathbf{u},
$$

and

$$
\begin{aligned}
\mathbf{G}_{\eta} & =\left(-\frac{\partial \eta}{\partial \bar{x}} \frac{\partial}{\partial \bar{y}}, 0\right) \\
L_{\eta} & =-\frac{\partial^{2} \eta}{\partial \bar{x}^{2}} \frac{\partial}{\partial \bar{y}}-2 \frac{\partial \eta}{\partial \bar{x}} \frac{\partial^{2}}{\partial \bar{x} \partial \bar{y}}+\left(\frac{\partial \eta}{\partial \bar{x}}\right)^{2} \frac{\partial^{2}}{\partial \bar{y}^{2}} \\
T_{\eta} & =-\frac{\partial \eta}{\partial \bar{t}} \frac{\partial}{\partial \bar{y}} .
\end{aligned}
$$

The discretization has been adapted from that used by Marquillie \& Ehrenstein $[19,20]$, for the simulation of a separated boundary-layer flow along a bump geometry, taking into account that in the present case the displacement $\eta(x, t)$ depends also on time. In the above equations the quantities have been made dimensionless using the displacement thickness $\delta_{0}^{*}$ of the Blasius profile that is chosen at inflow, and the uniform velocity $U_{\infty}^{*}$ far from the boundary layer. Fourth-order finite differences are used in the streamwise $x$ direction whereas the wall-normal $y$-direction is discretized using Chebyshevcollocation, an appropriate mapping (cf. [19]) transforming the unbounded domain into a finite domain $0 \leq \bar{y} \leq y_{\max }$. A value of $y_{\max }=80$ proved 
appropriate to recover uniform flow far from the wall. In all simulations 97 collocation points have been considered whereas a grid spacing $\Delta x=0.2$ has been used in the streamwise direction. For convenience the inflow is located at $x_{i}=0$ and domains with streamwise lengths from $x_{o} \approx 820$ to $x_{o} \approx 1200$ have been considered (and hence approximately 4000 to 6000 points in $x$ ). A semi-implicit scheme is used in time, considering the second-order accurate implicit backward differentiation formula for the Cartesian part of the diffusion term. The nonlinear and metric terms are evaluated using an explicit second-order Adams-Bashforth scheme. In most of the computations a step $\Delta t=10^{-2}$ has been considered for time-marching.

The wall displacement $\eta(x, t)$ is solution of the elastic plate equation

$$
m \frac{\partial^{2} \eta}{\partial t^{2}}+d \frac{\partial \eta}{\partial t}+B \frac{\partial^{4} \eta}{\partial x^{4}}+\kappa \eta=\sigma_{P}
$$

A spring stiffness term $\kappa$ has been added to model the action of a viscous substrate beneath the plate, similar to numerous previous studies dealing with stability issues (cf. for instance Carpenter \& Garrad [12]). The quantities $m$ and $B$ have been made dimensionless using the same reference length $\delta_{0}^{*}$ and time $\delta_{0}^{*} / U_{\infty}^{*}$ as for the Navier-Stokes system. They correspond respectively to the plate surface mass $h^{*} \rho_{P}^{*}$ and its bending stiffness $B^{*}=E^{*} h^{* 3} / 12\left(1-\nu^{2}\right)$, with $h^{*}$ and $\rho_{P}^{*}$ the plate thickness and density. In the dynamical equation (6), a viscous damping factor $d$ is added to the plate model. The finite-length plate is clamped into a rigid basis at both end positions $x_{a}$ and $x_{b}$ and the dynamical equation is completed by the clamped end-conditions

$$
\eta=\partial \eta / \partial x=0, \quad \text { at } \quad x=x_{a}, x_{b}
$$


The right-hand side

$$
\sigma_{p}=-p+\tau
$$

in the dynamical equation (6) stands for the wall-normal fluid-flow stress which is a function of the wall pressure $p$ and the normal projection of the viscous stress tensor

$$
\tau=(\mathbb{D} \cdot \mathbf{n}) \cdot \mathbf{n}=\frac{2}{R e}\left[\frac{\partial u}{\partial x} n_{x}^{2}+\left(\frac{\partial u}{\partial y}+\frac{\partial v}{\partial x}\right) n_{x} n_{y}+\frac{\partial v}{\partial y} n_{y}^{2}\right]
$$

with $\mathbf{n}=\left(n_{x}, n_{y}\right)$ the unit wall-normal vector.

In return, the plate motions affect the flow through the kinematic condition $v=\frac{d \eta}{d t}$. In the absence of wall longitudinal displacement, the latter condition becomes

$$
u=0 \quad v=\frac{\partial \eta}{\partial t}
$$

at the interface $\bar{y}=0$ in the transformed coordinate system.

Special care has been devoted to the computation of the pressure which is the dominant term in (7). The pressure is solution of the modified Poisson equation

$$
\bar{\nabla}^{2} p=-L_{\eta} p+2\left[\frac{\partial u}{\partial \bar{x}} \frac{\partial v}{\partial \bar{y}}-\frac{\partial u}{\partial \bar{y}} \frac{\partial v}{\partial \bar{x}}\right]
$$

and in order to achieve a full coupling between the flow field and the wall dynamics, the fractional time step procedure (cf. Kim [21]) is used. This approach has been adapted to the fluid flow computation in the time-dependent geometry resulting from the interaction with the compliant wall. Details concerning the time-marching and coupling procedure are provided in Appendix A. 


\subsection{Radiated noise model}

We intend to compute the radiated noise in the uniform flow domain from the acoustic sources resulting from the boundary-layer flow. In the present hydrodynamic context at a very low Mach number, noise computations are performed in the framework of the Lighthill's analogy, which is based on the assumption that the acoustic sources may be estimated from the boundary layer incompressible velocity fluctuation $\left(u^{\prime}, v^{\prime}\right)$. The dimensionless acoustic sources are

$$
\mathcal{S}\left(x_{1}, x_{2}\right)=\frac{\partial^{2} \mathcal{T}_{i j}}{\partial x_{i} \partial x_{j}}
$$

where the Lighthill's tensor

$$
\mathcal{T}_{i j}=u_{i}^{\prime} u_{j}^{\prime}-\tau_{i j}
$$

is a function of the viscous stress fluctuations tensor $\tau_{i j}$

$$
\tau_{i j}=\frac{1}{R e}\left(\frac{\partial u_{i}^{\prime}}{\partial x_{j}}+\frac{\partial u_{j}^{\prime}}{\partial x_{i}}\right) .
$$

In (10) the summation convention applies, by writing conveniently the coordinates $(x, y)$ as $\left(x_{1}, x_{2}\right)$ and the velocity fluctuations components $\left(u^{\prime}, v^{\prime}\right)$ as $\left(u_{1}^{\prime}, u_{2}^{\prime}\right)$. The acoustic pressure field in the boundary layer flow is then governed by the forced wave equation (cf. Lighthill [8])

$$
M^{2} \frac{\partial^{2} p^{\prime}}{\partial t^{2}}-\frac{\partial^{2} p^{\prime}}{\partial x^{2}}-\frac{\partial^{2} p^{\prime}}{\partial y^{2}}=\mathcal{S}(x, y, t)
$$

where $M$ is the flow Mach number. For the low Mach number considered here, convective effects due to the mean flow velocity are neglected in the acoustic equation. It is common (cf. Hariri \& Akylas [22], Dowling [10], Zheng [6], Shariff \& Wang [23] among many others) to employ the Green function 
approach to compute the acoustic pressure field. We introduce coordinates and time with capital letters and from the definition of the Dirac $\delta$-function we can write for the radiated pressure

$$
\begin{aligned}
p^{\prime}(X, Y, T) & =\int p^{\prime}(x, y, t) \delta(X-x, Y-y, T-t) d x d y d t \\
& =\int\left(M^{2} \frac{\partial^{2} G}{\partial t^{2}}-\nabla^{2} G\right) p^{\prime}(x, y, t) d x d y d t
\end{aligned}
$$

In the above equation the Green function $G(X, Y, T, x, y, t)$ is solution of

$$
M^{2} \frac{\partial^{2} G}{\partial t^{2}}-\nabla^{2} G=\delta(X-x, Y-y, T-t) .
$$

The domain of integration is sketched in figure 1. Integration by parts in (14) yields, by identifying the source term $\mathcal{S}$ from the pressure wave equation (13),

$$
\begin{aligned}
P(X, Y, T)= & \int G(X, Y, T, x, y, t) \mathcal{S}(x, y, t) d x d y d t \\
& +\int_{y=0}\left(\frac{\partial G}{\partial y} p_{w}(x, t)-G \frac{\partial p_{w}}{\partial y}(x, t)\right) d x d t
\end{aligned}
$$

(from now on we write the acoustic pressure field with capital letters $P(X, Y, T)$ ). Under the hypothesis of periodic flow in the streamwise $x$-direction as well as in time $t$ and assuming a decreasing wave-behaviour at infinity for the Green function, only the boundary term (16) along the plate is left. This term involves the flow perturbation pressure fluctuation $p_{w}(x, t)=p^{\prime}(x, 0, t)$ at the wall as well as its wall-normal derivative. The wall-displacement is small and the undisturbed wall position at $y=0$ is considered for the surface integral, which is a common assumption in noise prediction analyses in the presence of compliant surfaces (cf. Hariri \& Akylas [22]). The wall-normal pressure gradient $\partial p_{w} / \partial y$ is the sum of the wall displacement acceleration $\partial^{2} \eta / \partial t^{2}$, as a consequence of the kinematic condition on the wall 8 , and the 
nonlinear as well as viscous terms of the $y$-momentum equation at the wall. These nonlinear and viscous effects are generally discarded in the radiated pressure models based on the Lighthill's analogy, when making the assumption of a weak coupling between the wall and the flow dynamics. If the Green function is chosen such that

$$
\int_{y=0}\left(\frac{\partial G}{\partial y} p_{w}(x, t)-G \frac{\partial p_{w}}{\partial y}(x, t)\right) d x d t=0
$$

one recovers

$$
P(X, Y, T)=\int \frac{\partial^{2} G}{\partial x_{i} \partial x_{j}}\left(X, Y, T, x_{1}, x_{2}, t\right) \mathcal{T}_{i j}\left(x_{1}, x_{2}, t\right) d x_{1} d x_{2} d t
$$

with $\left(x_{1}, x_{2}\right)=(x, y)$ for convenience in the above equation. The partial derivatives have been switched from the Lighthill's volume source term (10) to the Green function by performing integration by parts and by assuming negligible viscous surface shear-stress fluctuations. When radiation due to viscous stress is taken into account, as for instance by Hu, Morfey \& Sandham [24] or Shariff \& Wang [23] for a turbulent boundary layer, the contribution

$$
P_{v}(X, Y, T)=-\int_{x_{2}=0} \frac{\partial G}{\partial x_{i}} \tau_{2 i}\left(x_{1}, 0, t\right) d x_{1} d t
$$

with $\tau_{i j}$ the fluctuation viscous stress tensor (12), is to be added. For the solution of the Green function, a Fourier transform in $x$ and $t$ is performed with

$$
\hat{G}(X, Y, T, k, y, \omega)=\int G(X, Y, T, x, y, t) e^{-i(k x-\omega t)} d x d t .
$$

Indeed, it will be shown in section 3.2 that the harmonically forced flow perturbation is nonlinearly saturated in space and time allowing for a Fourier analysis of the fluctuation flow quantities. 
In Appendix B it is shown how a Green function for the present problem can be determined. In the present boundary-layer configuration, the velocity fluctuations in the Lighthill's tensor $\mathcal{T}_{i j}$ become negligible for $y>h$ with $h$ of the order of the boundary-layer thickness. Consequently, the integration with respect to $x_{2}=y$ in (18) is performed to an upper limit $h$ at some units from the wall (the unit-length corresponding to the displacement thickness of the flow profile at inflow). The pressure radiation is evaluated for $Y \geq h$ and the expression (B.7) given in Appendix B for the Fourier transformed Green function has to be considered. Applying Parseval's formula and the product theorem for Fourier transforms one gets

$$
\begin{aligned}
& P(X, Y, T)= \\
= & \frac{e^{i \gamma Y}}{(2 \pi)^{2}} \int\left(\int_{0}^{h} \mathcal{L}_{+}(-k, y,-\omega) e^{i \gamma y} d y\right) \frac{i \gamma \hat{p}_{w}^{*}+\frac{\partial \hat{p}_{w}^{*}}{\partial y}}{2 i \gamma\left(i \gamma \hat{p}_{w}^{*}-\frac{\partial \hat{p}_{w}^{*}}{\partial y}\right)} e^{i(\omega T-K X)} d k d \omega \\
+ & \frac{e^{i \gamma Y}}{(2 \pi)^{2}} \int\left(\int_{0}^{h} \mathcal{L}_{-}(-k, y,-\omega) e^{-i \gamma y} d y\right) \frac{1}{2 i \gamma} e^{i(\omega T-K X)} d k d \omega
\end{aligned}
$$

where

$$
\mathcal{L}_{ \pm}(k, y, \omega)=\left[k \delta_{i 1} \pm \gamma \delta_{i 2}\right]\left[k \delta_{j 1} \pm \gamma \delta_{j 2}\right] \hat{\mathcal{T}}_{i j}(k, y, \omega)
$$

with $\hat{\mathcal{T}}_{i j}(k, y, \omega)$ the Fourier transform of the Lighthill's tensor $\left(\delta_{i j}\right.$ being the Kronecker-symbol). Finally, from (21) it follows that the Fourier transform of $P(X, Y, T)$ can be written as a function of the Fourier transforms of the wall pressure, the wall-normal pressure gradient and the Lighthill's stress 
tensor with

$$
\begin{aligned}
\hat{P}(k, Y, \omega) & =\frac{e^{i \gamma Y}\left(i \gamma \hat{p}_{w}+\frac{\partial \hat{p}_{w}}{\partial y}\right)}{2 i \gamma\left(i \gamma \hat{p}_{w}-\frac{\partial \hat{p}_{w}}{\partial y}\right)} \int_{0}^{h} \mathcal{L}_{+}(k, y, \omega) e^{i \gamma y} d y \\
& +\frac{e^{i \gamma Y}}{2 i \gamma} \int_{0}^{h} \mathcal{L}_{-}(k, y, \omega) e^{-i \gamma y} d y .
\end{aligned}
$$

The root $\gamma$ of $\gamma^{2}=M^{2} \omega^{2}-k^{2}$ is chosen according to the radiation condition (B.5) at infinity (cf. Appendix B). In this expression the contribution due to wall shear stress fluctuations has been discarded, similarly to the analyses by Zheng [6] or Dowling [10]. To assess the validity of this hypothesis, according to equation (19) the Fourier transformed quantity can be computed with

$$
\hat{P}_{v}(k, Y, \omega)=\frac{\left(i k \hat{p}_{w} \hat{\tau}_{21}(k, 0, \omega)-\frac{\partial \hat{p}_{w}}{\partial y} \hat{\tau}_{22}(k, 0, \omega)\right) e^{i \gamma Y}}{\frac{\partial \hat{p}_{w}}{\partial y}-i \gamma \hat{p}_{w}}
$$

(where $\hat{\tau}_{i j}(k, 0, \omega)$ is the Fourier transformed viscous stress tensor at the wall). This issue will be addressed in section 4.2).

\section{Fluid-structure dynamics}

Elastic plates with different characteristics have been considered, in particular in terms of natural plate frequencies and stiffness. Parameter values have been chosen, typical of polyurethane and glass-resins composite which are used for instance in the manufacturing of sonar flank arrays that equip underwater vehicles. Results for two plates are reported. The plate called plate 1 has a non zero stiffness parameter (accounting for a spring-backed plate) and its natural frequencies are distinct from that of the harmonic fluid 


\begin{tabular}{|c|c|c|}
\hline Dimensionless parameters & Plate 1 & Plate 2 \\
\hline thickness $h$ & 26.3 & 14.9 \\
\hline mass $m$ & 28.2 & 26.2 \\
\hline bending stiffness $B$ & $7.8510^{5}$ & $1.5810^{7}$ \\
\hline stiffness $\kappa$ & 4.58 & 0.0 \\
\hline \multicolumn{3}{|l|}{ Dimensional parameters } \\
\hline thickness $h^{*}$ & $4 \mathrm{~mm}$ & $2.275 \mathrm{~mm}$ \\
\hline density $\rho_{P}^{*}$ & $1100 \mathrm{~kg} \cdot \mathrm{m}^{-3}$ & $1800 \mathrm{~kg} \cdot \mathrm{m}^{-3}$ \\
\hline Young's modulus $E^{*}$ & $1.3110^{8} \mathrm{~Pa}$ & $1.4310^{10} \mathrm{~Pa}$ \\
\hline Poisson's ratio $\nu_{P}$ & 0.49 & 0.49 \\
\hline stiffness $\kappa^{*}$ & $10^{10} \mathrm{~kg} \cdot \mathrm{m}^{-2} \cdot \mathrm{s}^{-2}$ & 0.0 \\
\hline
\end{tabular}

Table 1: Characteristics of the two elastic plates considered in the study.

flow forcing. Plate 2 has lower natural frequencies and zero stiffness. The Reynolds number $R e=2000$, based on the displacement thickness of the Blasius profile at inflow, has been considered which is much higher than the critical Reynolds number $R e_{c} \approx 520$ for convective flow instabilities. The dimensionless as well as physical parameters, considering $\delta_{a}^{*}=\operatorname{Re} \nu / U_{\infty}^{*}$ with $\nu$ the kinematic viscosity of water and $U_{\infty}^{*}=18 \mathrm{~m} / \mathrm{s}$, are reported in table 1.

\subsection{Steady state and added mass phenomena}

In the rigid-wall case the flow is known to be convectively unstable and in the absence of external perturbations the basic state is the non-parallel 
boundary-layer flow $\mathbf{U}=(U, V)$ which can be reached by time-marching. The dashed line in figure 3 depicts the pressure distribution along the wall in the rigid case. The pressure has been set to zero at inflow $(x=0, y=0)$ and is shown to exhibit a small negative gradient along the wall. Notice that this solution corresponds to a steady state of the Navier-Stokes system and there is no reason for the pressure to be constant, as it would be when using Prandtl's boundary-layer approximation.

For the flow along the elastic plate, steady flow states are retrieved by adding high damping into the dynamical equation (6). Plates of dimensionless length $L=614\left(L^{*} \approx 9.3 \mathrm{~cm}\right)$, clamped between $x_{a}=40$ and $x_{b}=654$ into a rigid wall upstream and downstream, have been considered. The computational domain in the streamwise direction is $0 \leq x \leq 820$.

The coupling leads to a bent steady state, the resulting plate shape depending on the plate characteristics, as shown in figure 2. To interpret the wall behaviour, it is convenient to solve equation (6) by use of an expansion into wall modes

$$
\eta(x, t)=\sum_{j=1}^{N} a_{j}(t) \hat{\eta}_{j}(x),
$$

where the modes $\hat{\eta}_{j}(x)$ are solutions of the eigenvalue problem

$$
B \frac{d^{4} \hat{\eta}_{j}(x)}{d x^{4}}+\kappa \hat{\eta}_{j}(x)=\lambda_{j} \hat{\eta}_{j}(x)
$$

The fourth-order differential operator in (26) is discretized using secondorder centered finite differences. The clamped boundary conditions are added and the modes and the eigenvalues are computed by solving the algebraic eigenvalue problem. The dynamical equation (6) is then turned into a set of 


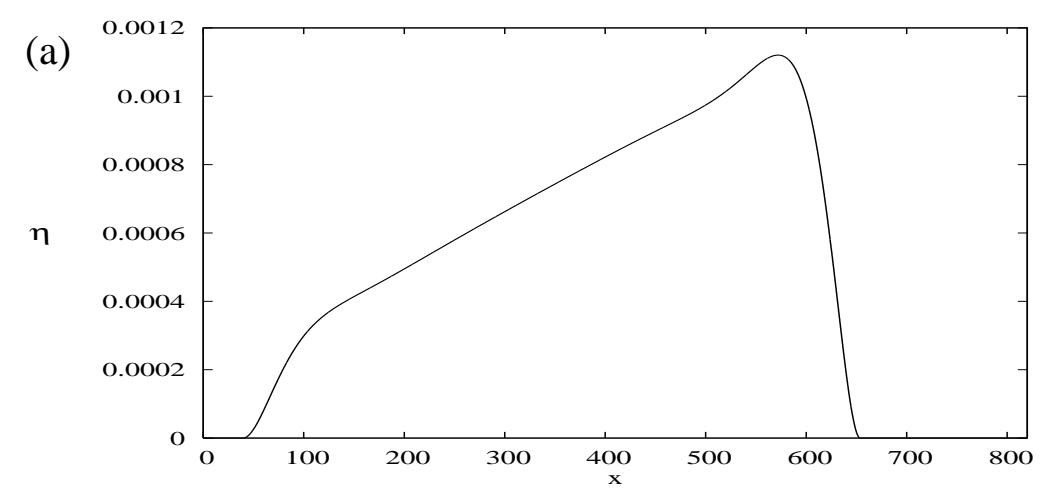

(b)

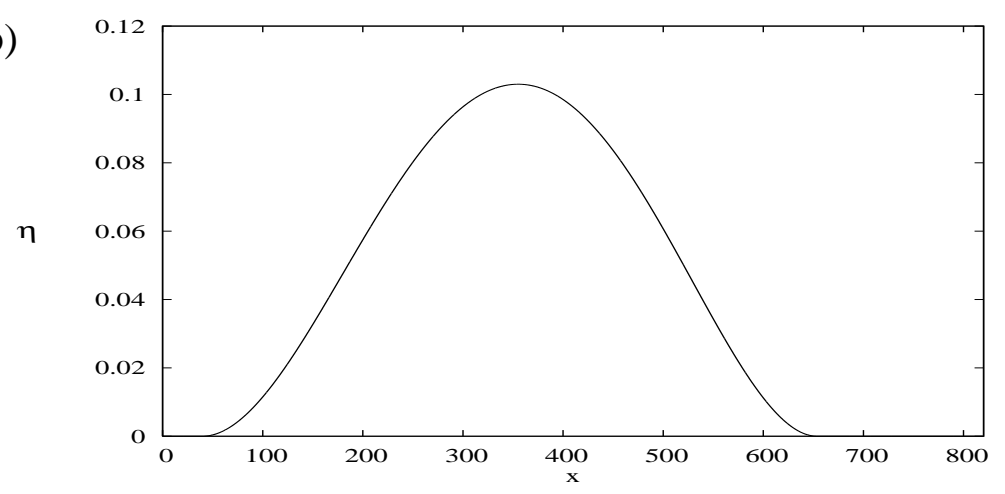

Figure 2: Steady-state displacements (a) plate 1 (b) plate 2.

equations

$$
m \frac{d^{2} a_{j}(t)}{d t^{2}}+d_{j} \frac{d a_{j}(t)}{d t}+\lambda_{j} a_{j}(t)=\left\langle\sigma_{P}, \hat{\eta}_{j}\right\rangle
$$

whose solutions are the modal amplitudes $a_{j}$. Due to the orthogonality of the modes, the projection $\left\langle\sigma_{P}, \hat{\eta}_{j}\right\rangle$ is merely the discrete inner product with the modes. It has been checked that the truncation $N=240$ used in the expansion (25) is high enough to capture all significant spatial scales. The resulting wall displacement is hence equivalent to that recovered when solving directly (6). In the case of plate 1, it appears that for the first modes the stiffness dominates and $\lambda_{j} \approx \kappa$. The wall displacement at the steady state is shown in figure 2 (a). The wall shape is seen to increase monotonously in a region 


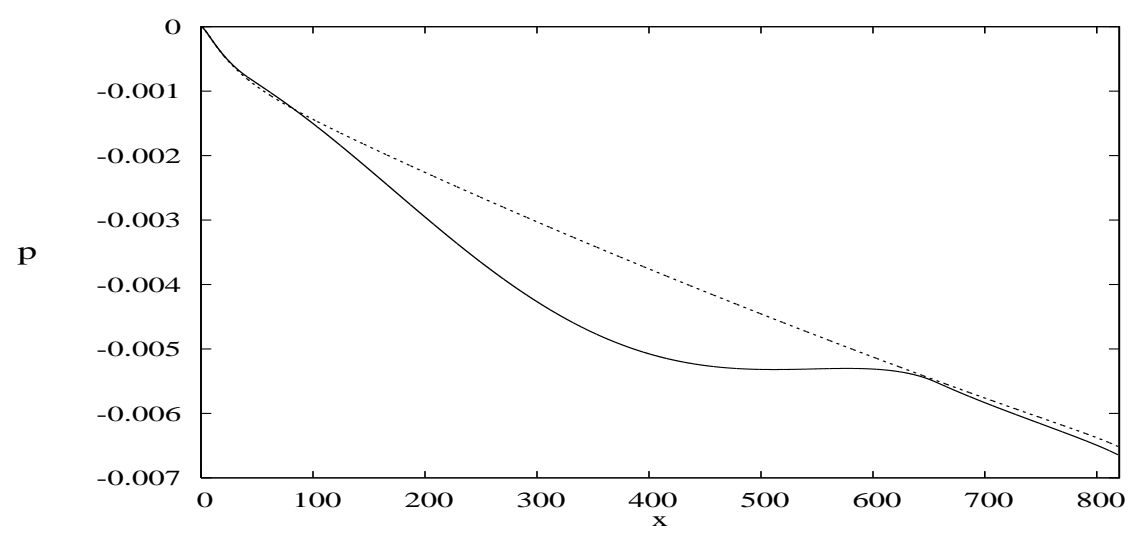

Figure 3: Steady state pressure spatial distributions along the rigid wall $(\cdots)$ and plate 2 $(-)$.

between the leading and trailing edges, while exhibiting sharp gradients at both edges due to the clamped end conditions. The corresponding pressure distribution, not shown, is very close to that along the rigid wall, depicted as the dashed curve in figure 3 . For plate 2 however, the eigenvalue of the first mode dominates, leading to the distribution shown in figure 2 (b). Now the plate displacement is much higher, leading to a significant modification of the steady-state pressure along the plate, which is shown as the solid line in figure 3.

Once a steady state along the compliant wall is obtained, the damping factor is decreased generating transient oscillations, in the absence of external perturbations. The natural frequencies of the system (27) can easily be seen to be $\omega_{j}=\sqrt{\lambda_{j} / m}$ and the damping factor can conveniently be chosen for each mode as $d_{j}=2 m \omega_{j} \varepsilon_{j}$, so that a low $\varepsilon_{j}$ value gives rise to a decreased frequency $\omega_{j} \sqrt{1-\varepsilon_{j}^{2}}$ and to an exponentially decreasing amplitude factor $e^{-\varepsilon_{j} \omega_{j} t}$. A damping factor of $\varepsilon_{j} \approx 0.05$ proved to be sufficient to avoid 


\begin{tabular}{ccccc}
\hline & \multicolumn{2}{c}{ Plate 1} & \multicolumn{2}{c}{ Plate 2 } \\
\hline$j$ & $\omega_{j}$ & $\omega_{j}^{\prime}$ & $\omega_{j}$ & $\omega_{j}^{\prime}$ \\
\hline 1 & 0.40 & 0.26 & 0.05 & - \\
2 & 0.40 & 0.26 & 0.13 & 0.09 \\
3 & 0.41 & 0.26 & 0.25 & 0.16 \\
4 & 0.41 & 0.26 & 0.41 & 0.27 \\
5 & 0.42 & 0.26 & 0.61 & 0.42 \\
\hline
\end{tabular}

Table 2: Initial natural frequencies $\omega_{j}$ and frequencies $\omega_{j}^{\prime}$ with added mass for the two elastic plates.

resonant plate dynamics through coupling with the fluid wall pressure when considering plate 1 . In the case of plate 2 without stiffness, given the large scale plate deformation shown in figure 2 (b), it was necessary to completely damp out the first mode in order to avoid high-amplitude oscillations at the scale of the computational domain. The value $\varepsilon_{j} \approx 0.05$ proved to be sufficient for the higher modes $j=2,3, \cdots$. In the system $(27)$, the dominant term of the right-hand side is the projection of the wall pressure, in comparison with the viscous stress term which is small at the high Reynolds number considered. The projection of the wall pressure onto a mode shape has an in-phase temporal evolution with respect to the mode amplitude $a_{j}$, due to the added mass effect, that is

$$
\left\langle-p, \hat{\eta}_{j}\right\rangle=\beta_{j} a_{j}+\gamma_{j}
$$

The added mass factors $\beta_{j}$ have been numerically determined for the first modes and the modified frequencies $\omega_{j}^{\prime}=\sqrt{\left(\lambda_{j}-\beta_{j}\right) / m}$ appeared to differ 
by about $30 \%$ from the natural ones. Table 2 summarizes the natural as well as the modified frequencies for the two plates, considering the first 5 modes. Note that as we mentioned before, the frequencies of the first modes for plate 1 are almost identical, due to the dominant stiffness parameter $\kappa$ in that case.

\subsection{Unsteady fluid-structure coupling}

In order to trigger the flow instability, a volume forcing is introduced in the vicinity of the inflow, located at $x_{i}=0$. The forcing functions $f_{u}, f_{v}$ in the streamwise and wall-normal directions respectively are

$$
\begin{aligned}
& f_{u}(t)=-A\left(y-y_{f}\right) \exp \left(\frac{-\left(x-x_{f}\right)^{2}}{2 \sigma_{x}^{2}}-\frac{\left(y-y_{f}\right)^{2}}{2 \sigma_{y}^{2}}\right) \Sigma_{i} \cos \left(\omega_{i} t\right) \\
& f_{v}(t)=A \frac{\sigma_{y}^{2}}{\sigma_{x}^{2}}\left(x-x_{f}\right) \exp \left(\frac{-\left(x-x_{f}\right)^{2}}{2 \sigma_{x}^{2}}-\frac{\left(y-y_{f}\right)^{2}}{2 \sigma_{y}^{2}}\right) \Sigma_{i} \cos \left(\omega_{i} t\right)
\end{aligned}
$$

This volume forcing, which is oscillatory in time at various frequencies $\omega_{i}$, is set divergence-free with an appropriate Gaussian-type spatial envelope. The parameters $\left(x_{f}, y_{f}\right)=(35,1.5)$ fix the location of the forcing in space, whereas $\left(\sigma_{x}, \sigma_{y}\right)=(2,0.3)$ account for its spatial extent which is about 10 in the streamwise direction and 1.5 in the wall-normal direction. The parameter $A$ characterizes the forcing amplitude, such that the maxima of $\left|f_{u}\right|$ and $\left|f_{v}\right|$ are respectively approximately $0.18 \mathrm{~A}$ and $0.03 \mathrm{~A}$. Four forcing frequencies have been considered with $\omega_{1}=0.04, \omega_{2}=0.08, \omega_{3}=0.12, \omega_{4}=0.16$. Note that the first three frequencies are in the unstable frequency range for the Blasius profile at inflow, at the Reynolds number $R e=2000$ considered. The harmonic forcing gives rise to a wavepacket which grows along the plate due to the convective instability. When the permanent flow regime is reached, 


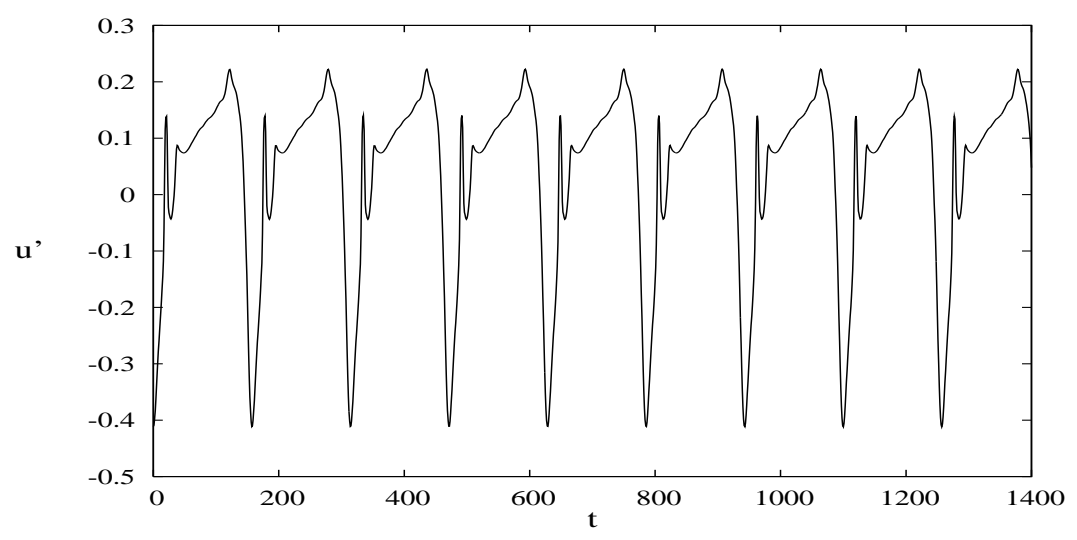

Figure 4: Steady state streamwise velocity for the flow bounded by the rigid wall at $x=450$ and $y=0.63$, for $A=0.1$.

the flow perturbation amplitude saturates at some distance from the forcing, as a consequence of the nonlinearities in the Navier-Stokes system.

First, the flow perturbation has been computed in the rigid-plate case for a small forcing amplitude $A=0.005$ and a high one $A=0.1$. A permanent perturbed flow regime sets in when the convective instability reaches the outflow boundary (at $t \approx 1800$ for $x_{o}=820$ and $A=0.1$ ). Given the harmonic nature of the forcing, the resulting disturbance flow quantities exhibit a timeperiodic behaviour, as shown in figure 4 for the streamwise component $u^{\prime}$ of the perturbation flow velocity $\mathbf{u}^{\prime}=\mathbf{u}-\mathbf{U}$, with $\mathbf{U}$ the unperturbed steady state (the time $t$ in the figure is reset to zero when the permanent flow regime is reached). Figure 5 shows the instantaneous streamwise component of the perturbation flow velocity at the distance $y=0.63$ from the wall, for the two amplitudes $A=0.005,0.1$ which have been considered. These spatial distributions are plotted slightly downstream of the forcing position $x=35$, from $x=100$ to $x=800$. While for the low amplitude forcing there is a con- 
(a)

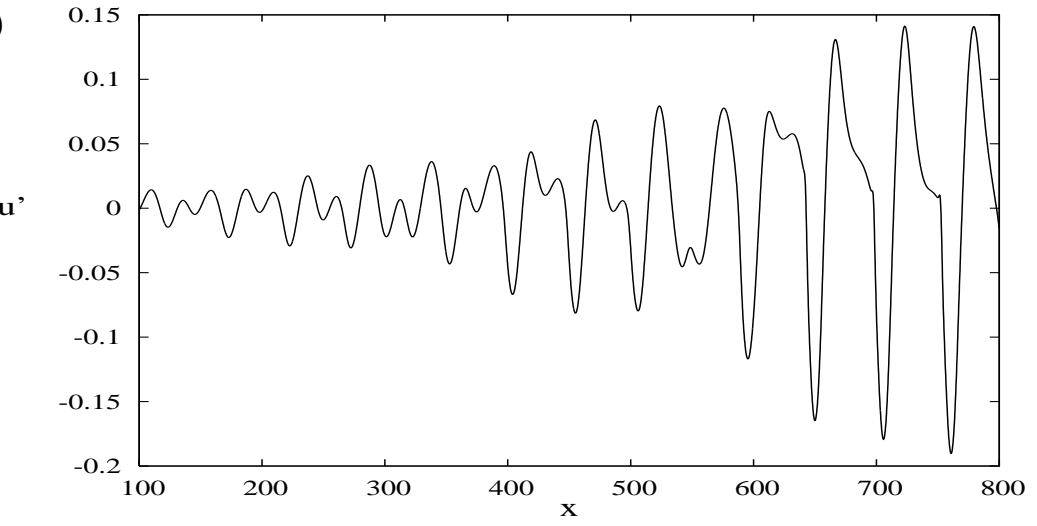

(b)

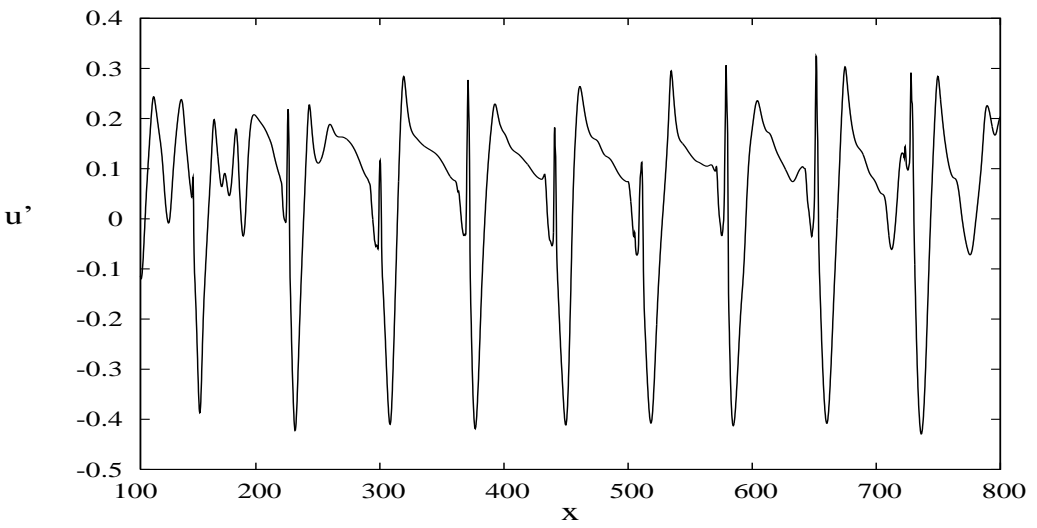

Figure 5: Instantaneous spatial distributions of the perturbation velocity component $u^{\prime}$ at $y=0.63$ for the flow bounded by the rigid wall, for two forcing amplitudes (a) $A=0.005$ (b) $A=0.1$.

tinuous perturbation growth exhibiting saturation only close to the outflow boundary, the flow perturbation is seen to saturate already at $x=100$ when the high amplitude is considered.

One key quantity in the forthcoming analysis is the perturbation pressure along the wall, whose spatial evolution is shown in figure 6. Again, there is clear evidence of saturation at $x=100$ for the high-amplitude forcing with $A=0.1$ (figure $6(\mathrm{~b})$ ). 


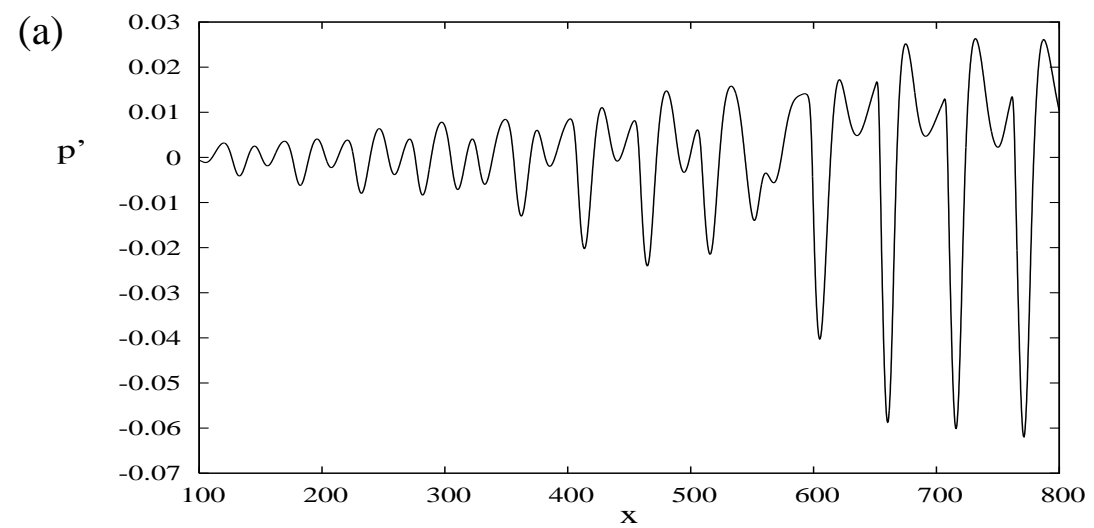

(b)

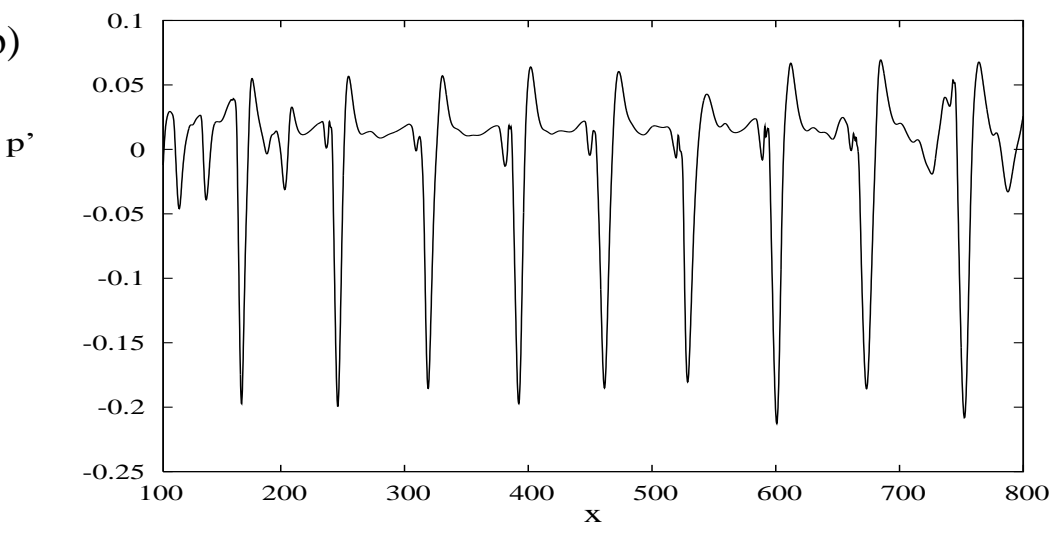

Figure 6: Instantaneous spatial distributions of the wall pressure fluctuation for the flow bounded by the rigid wall, for two forcing amplitudes (a) $A=0.005$ (b) $A=0.1$. 
Although the simulations have been performed for the finite-length domain $0 \leq x \leq 820$, the flow perturbation is seen to exhibit almost periodic structures in time as well as in space, for the high-amplitude forcing and inside the box $100 \leq x \leq 800$ in the streamwise direction. Hence, a wavenumber-frequency Fourier transform of the flow quantities appears to be legitimate when the forcing amplitude $A=0.1$ is considered. It is defined, for instance for the perturbation pressure at the wall, as

$$
\hat{p}(k, y=0, \omega)=\int_{t_{0}}^{t_{0}+T} \int_{l_{0}}^{l_{0}+L} p^{\prime}(x, 0, t) e^{-i(k x-\omega t)} d x d t
$$

with $T=2048$ and $L=700\left(l_{0}=100\right)$. For that purpose the pressure has been sampled at every time unit and every discretization point in the streamwise $x$-direction. Note that the rectangular window has been considered for the spectral analyses and the Fortran 90 fftpackage has been used which is not restricted to a power of 2 number of data.

The transformed wall perturbation pressure is depicted in figure 7 . The structure is characteristic of a convective wave in the downstream direction and it is seen to be dominated by peaks at the four forcing frequencies, as well as their harmonics which are generated by nonlinearities in the flow. A convective disturbance velocity close to 0.5 can be estimated from the data.

For the same forcing device and amplitudes, the flow instabilities have been computed for the coupled fluid-structure system, with elastic plates of length 614 (clamped at $x_{a}=40$ and $x_{b}=654$ ) for an overall domain extending from $x_{i}=0$ to $x_{o}=820$. The resulting instantaneous wall displacement for plate 1 is depicted in figure 8 , for both amplitudes $A=0.005$ and $A=0.1$. It exhibits a fluctuation around the steady state deformation (shown in figure 2 (a)) which increases with the amplitude. The time-dependent wall fluctu- 


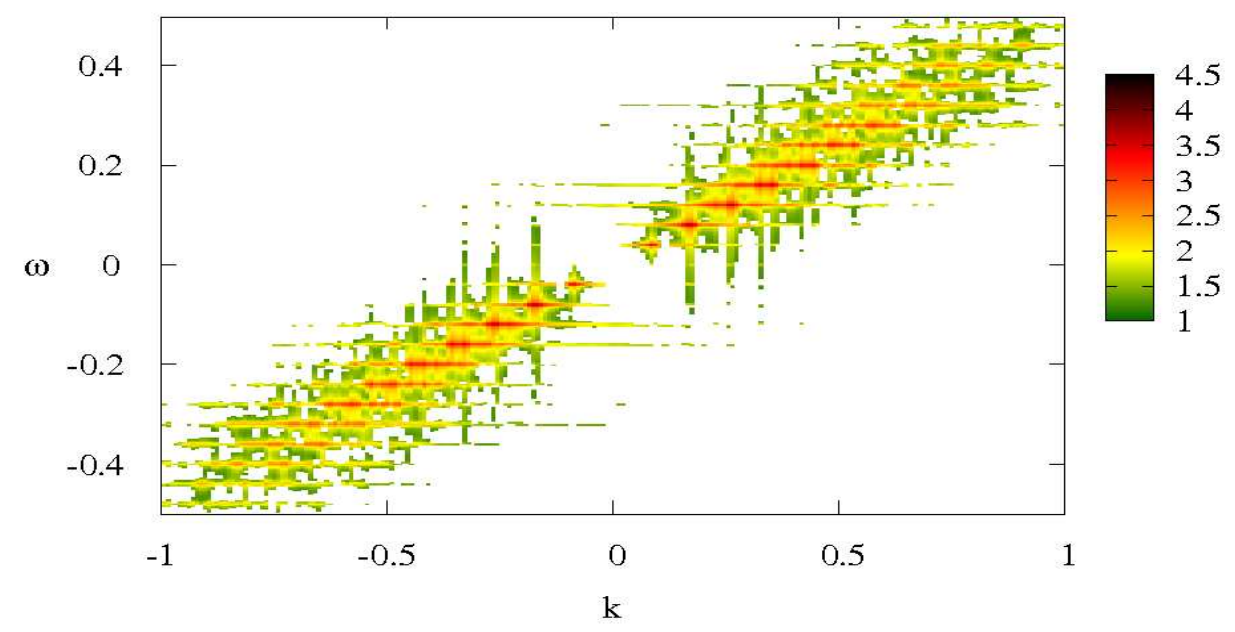

Figure 7: $\log |\hat{p}(k, y=0, \omega)|$ for the rigid wall with $A=0.1$, color scale ranging from 1 to 4.5 .

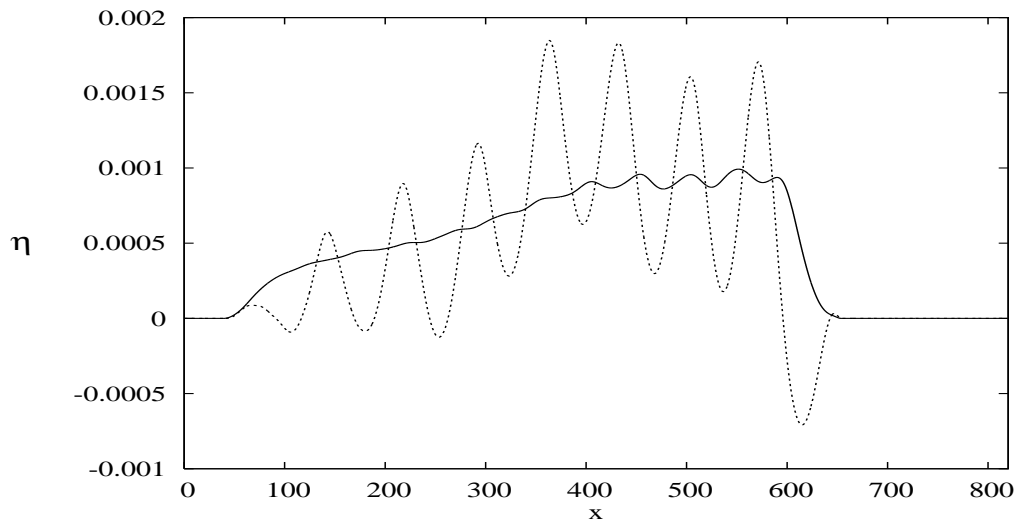

Figure 8: Instantaneous spatial evolutions of plate 1 displacements for $A=0.005(-)$ and $A=0.1(\cdots)$. 


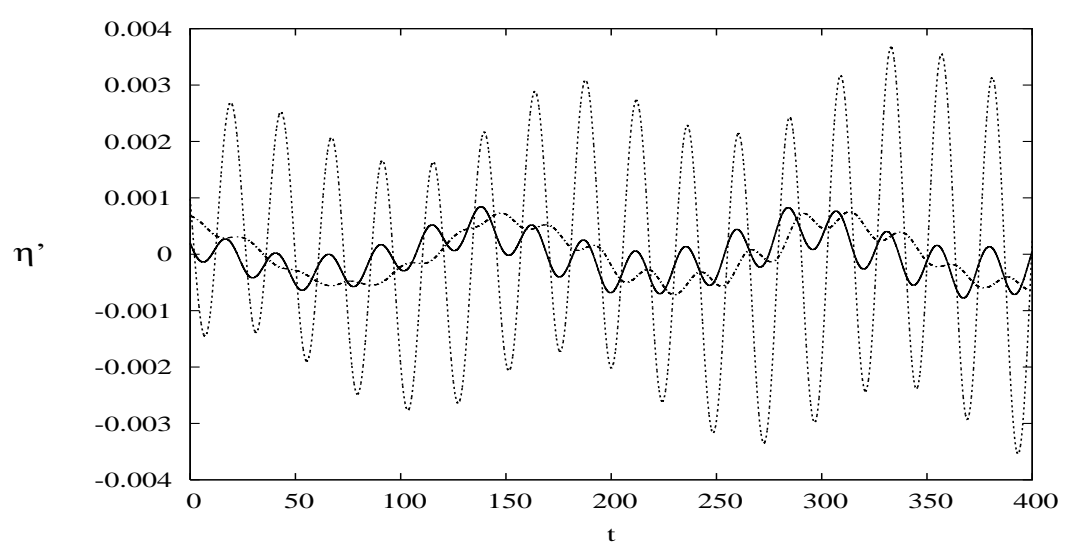

Figure 9: Plate 1 displacement fluctuations $\eta^{\prime}=\eta-\bar{\eta}$ at $x=194(-), x=347(-\cdot-)$ and $x=500(\cdots)$ for $A=0.1$.

ations $\eta^{\prime}=\eta-\bar{\eta}$, with $\bar{\eta}$ the time-averaged value, at locations $x=194$, 347 and 500 , which correspond to $1 / 4,1 / 2$ and $3 / 4$ of the plate length, are shown in figure 9 , for $A=0.1$. There is clear evidence of a main fluctuation at the modal frequency $\omega^{\prime}=0.26$ (taking into account the added mass, cf. table 2) and a modulation at a lower frequency $\omega \approx 0.04$ associated with the most unstable convective flow instability. Note that given the particular instantaneous wall shape, the amplitude of the fluctuation increases along the plate, while being almost saturated in time at a given location.

The instability behaviour for plate 2 is very different, as shown in figure 10 , where the instantaneous wall fluctuation shape is depicted for $A=0.005$ and $A=0.1$. The main deformation is seen to be associated with mode 3. Indeed, in the previous section (cf. table 2), the modified frequency of this mode has been shown to be $\omega^{\prime}=0.16$, which is precisely one of the forcing frequencies for the flow. The wall fluctuation at mid-plate is shown in figure 11 confirming the oscillation at a frequency close to 0.16 . For the 


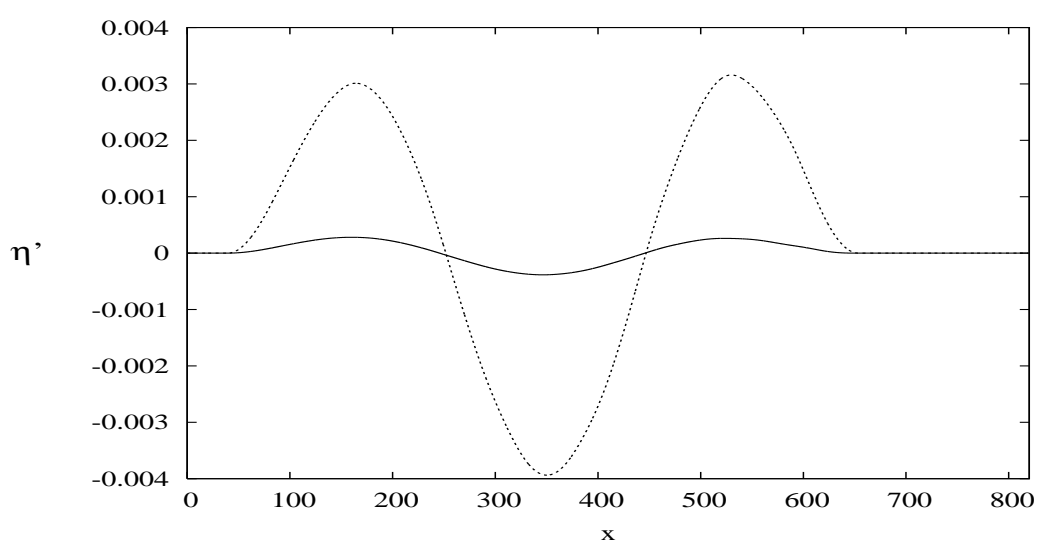

Figure 10: Instantaneous spatial evolutions of plate 2 displacement fluctuations $\eta^{\prime}=\eta-\bar{\eta}$ for $A=0.005$ (一) and $A=0.1(\cdots)$.

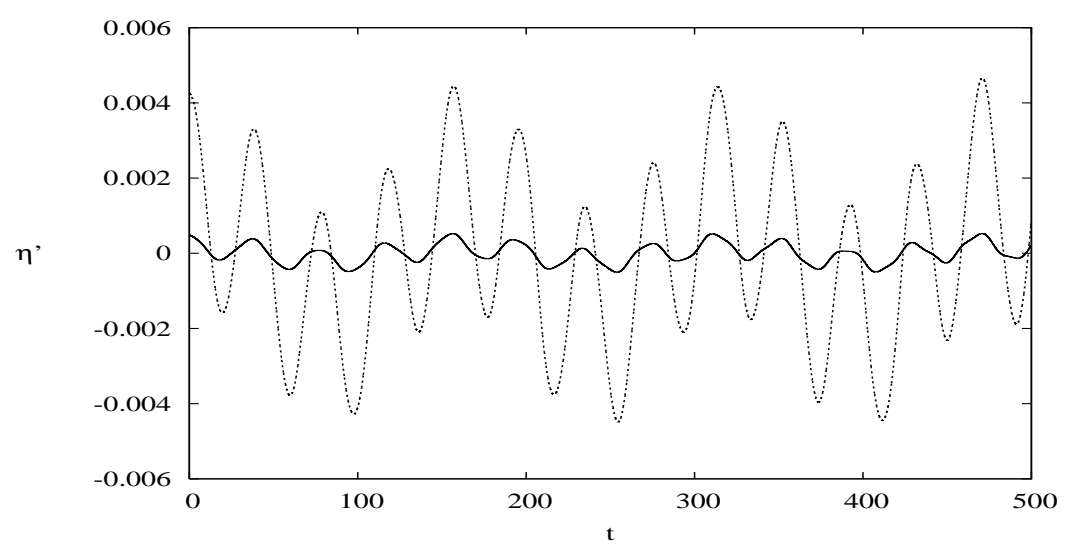

Figure 11: Plate 2 displacement fluctuations $\eta^{\prime}=\eta-\bar{\eta}$ at mid-plate for $A=0.005(-)$ and $A=0.1(\cdots)$. 


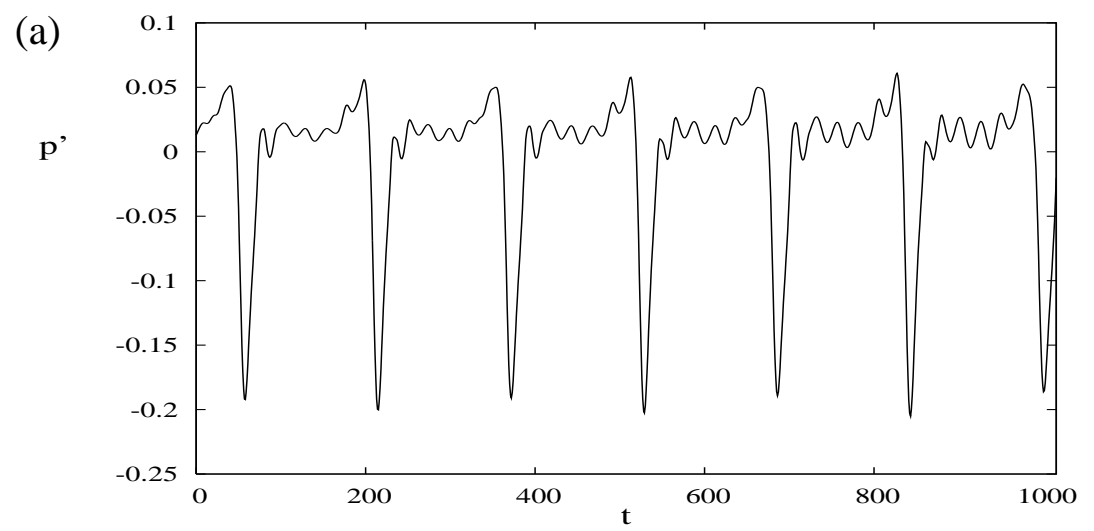

(b)

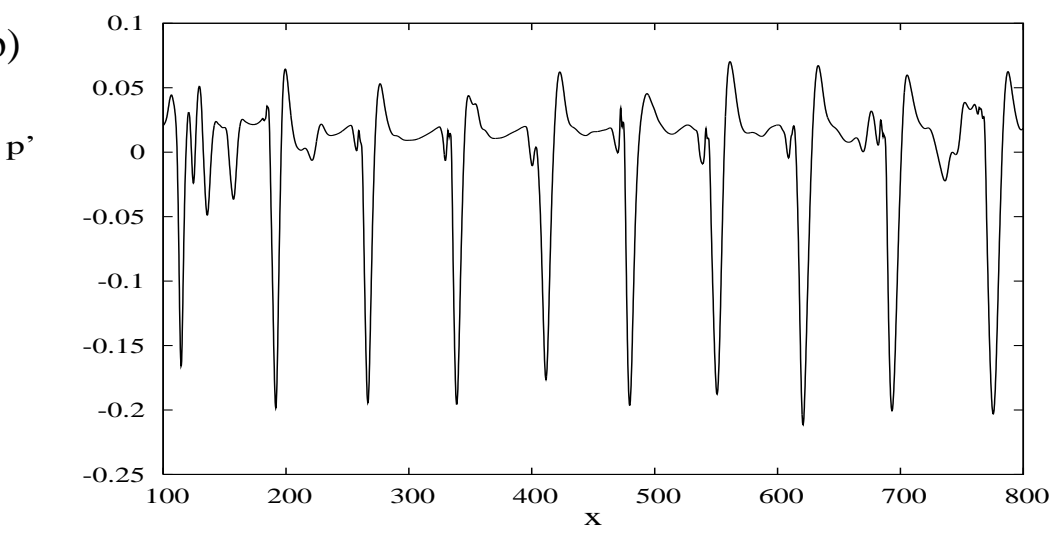

Figure 12: Plate 1 wall pressure fluctuation for $A=0.1$ : (a) time evolution at $x=450$ (b) instantaneous spatial distribution.

flow perturbation with high amplitude there is again a modulation in the wall displacement associated with the convective flow frequency of 0.04 .

The perturbation pressure along plate 1 is shown in figure 12 for the high-amplitude forcing $A=0.1$. The wall pressure fluctuation at $x=450$ is depicted as a function of time as well as the instantaneous pressure along the compliant wall for $100 \leq x \leq 800$. Similarly to the rigid case, the perturbation structure is saturated in time and space, for plate 1 as well as plate 2 (these latter results are not shown). 
Again, a wavenumber-frequency Fourier transform can be performed and the results are shown in figure 13 for both plates. While the convective part of the transformed pressure is very similar to that of the rigid case (cf. figure 7), additional peaks are clearly visible at $\omega= \pm 0.26$ in figure 13 (a) for plate 1 , with $k$ close to zero, corresponding to the dominant mode frequency. The Fourier transform for plate 2 exhibits additional peaks for $k \approx 0$ at $\omega= \pm 0.16$ but interestingly also at $\omega= \pm 0.42$, which is actually the frequency (modified by added mass) of the fifth mode given in table 2 .

The wavenumber Fourier transform at constant $\omega=0.26$ for plate 1 is shown in figure 14 and is compared with the rigid wall result. The convective peak (due to the flow perturbation harmonics) is visible at $k \approx 0.52$ together with the modal peak at $k \approx 0$. The latter peak is proper to trigger acoustic pressure fluctuations to be addressed in the next section.

Considering the peak at $\omega=0.42$ for plate 2 , the corresponding cut as a function of the wavenumber $k$ is shown in figure 15. Now, the convective peak is located at $k \approx 0.75$, however with a lower amplitude, and again the modal peak can be distinguished for vanishing wavenumbers.

\section{Radiated sound}

The acoustic pressure field is determined as solution of the equation (13) for the forcing associated with the Lighthill's tensor $\mathcal{T}_{i j}=u_{i}^{\prime} u_{j}^{\prime}-\tau_{i j}$, with $\tau_{i j}$ the viscous fluctuation stress tensor (12). In section 2.2 the coordinates $(X, Y, T)$ have been introduced for the acoustic pressure $P(X, Y, T)$, whose expression using the Green function approach is given by (18). By assuming homogeneity in the streamwise direction as well as statistically stationary 


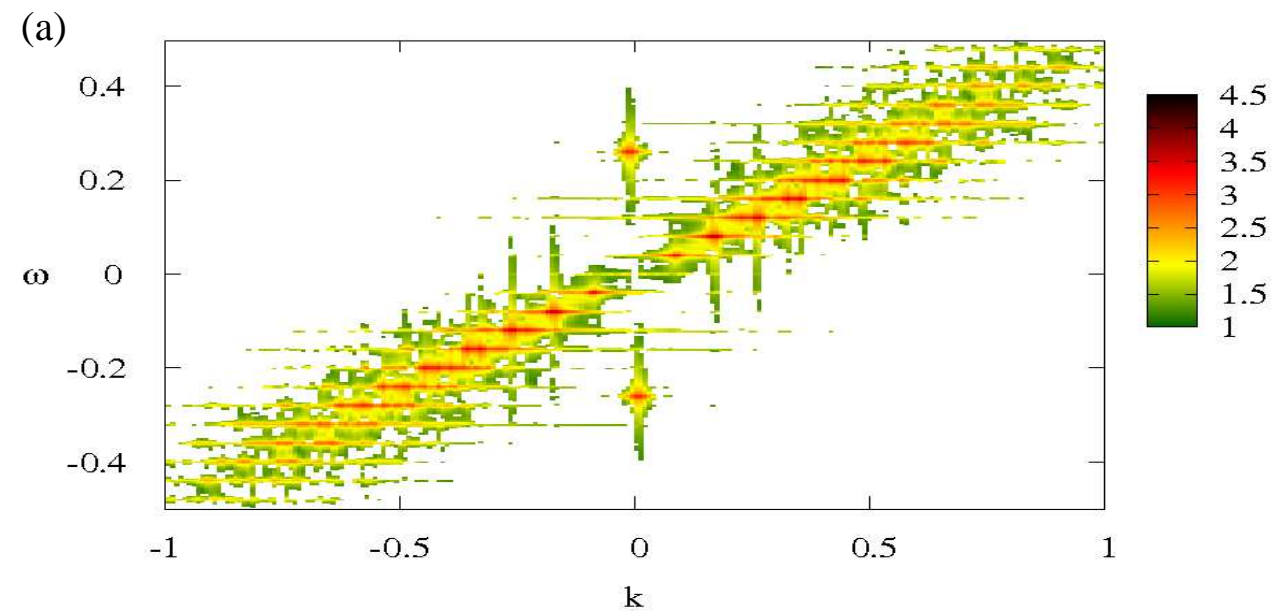

(b)

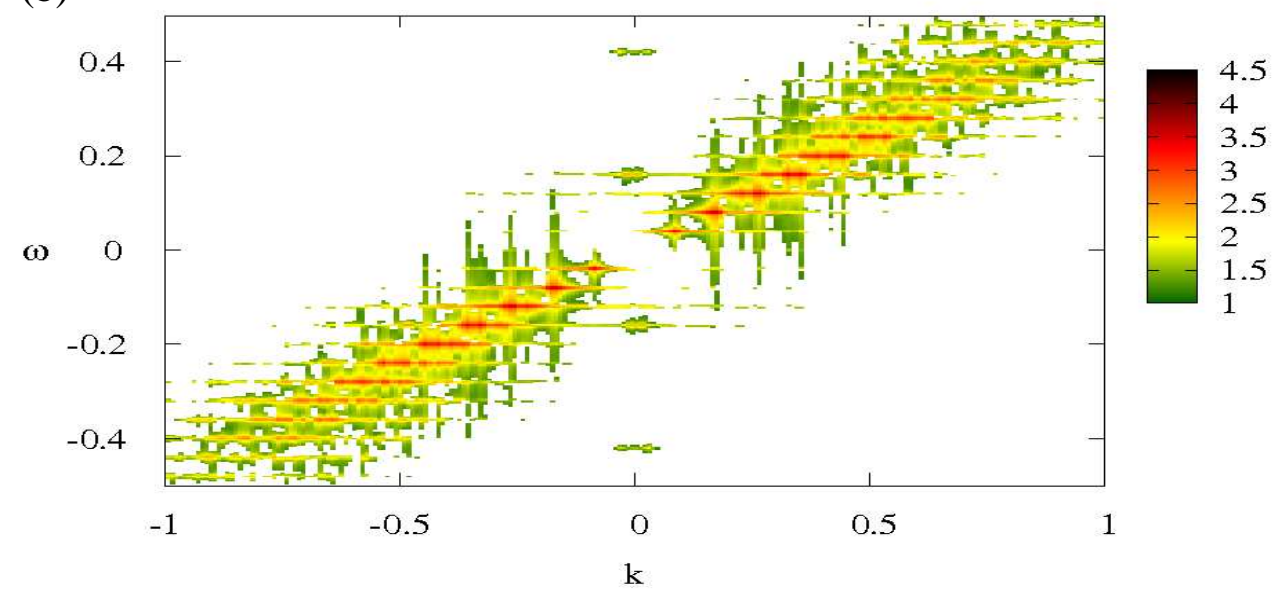

Figure 13: $\log |\hat{p}(k, y=0, \omega)|$ for $A=0.1$ and (a) plate 1, (b) plate 2 , color scales ranging from 1 to 4.5 . 


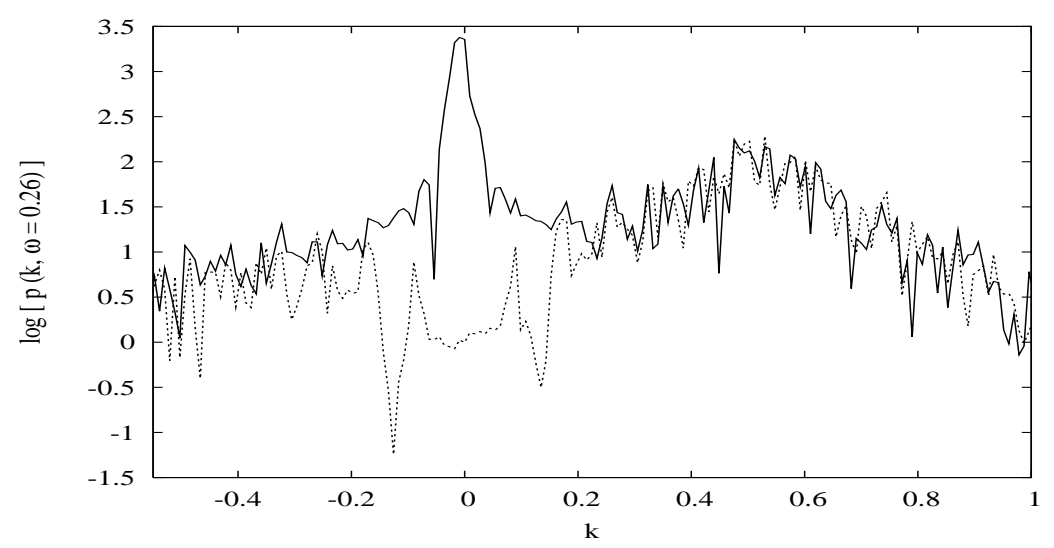

Figure 14: Comparison of the spectral components $\log |\hat{p}(k, y=0, \omega=0.26)|$ for plate 1 $(-)$ and the rigid wall $(\cdots)$ with $\mathrm{A}=0.1$.

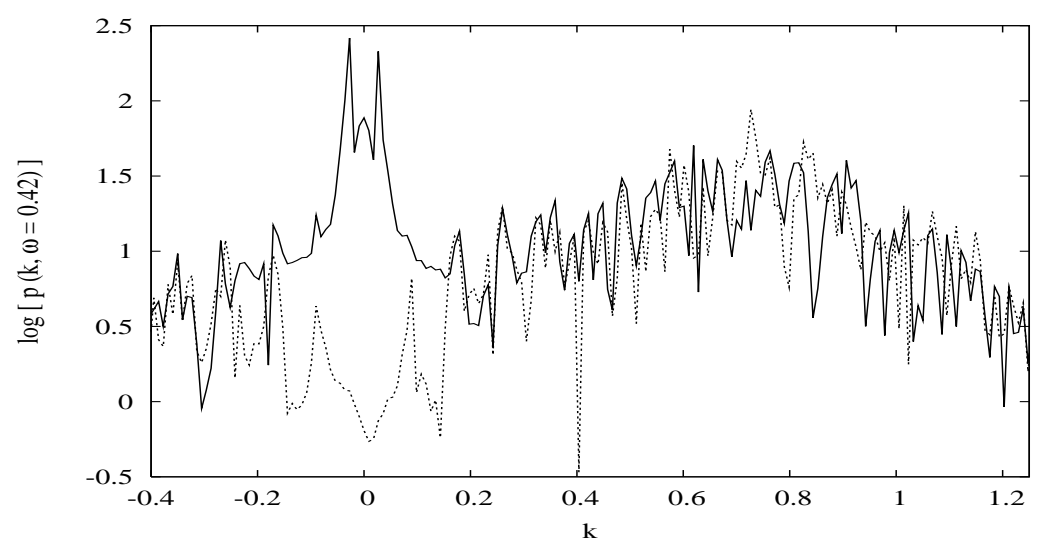

Figure 15: Comparison of the spectral components $\log |\hat{p}(k, y=0, \omega=0.42)|$ for plate 2 $(-)$ and the rigid wall $(\cdots)$ with $\mathrm{A}=0.1$. 
behaviour in time, in wall bounded turbulent flow it is common to compute the Fourier transformed acoustic pressure. Indeed, in general the spectral response of the perturbation flow quantities are available rather than the physical quantities in space and time (which would represent a tremendous amount of data). Our simulation data have been shown to be saturated in time as well as over a large distance in the streamwise direction, for the nonlinear perturbation evolution with forcing amplitude $A=0.1$. Hence, for this high-amplitude flow forcing a periodic perturbation flow assumption in the streamwise direction and in time has been adopted and the Fourier transformed acoustic pressure $\hat{P}(k, Y, \omega)$ given by formula (23) is computed. The flow quantities forming the Lighthill's tensor have been sampled and stored at the discrete points in $y$ inside the boundary-layer, over a time interval $T=2048$ in the permanent flow regime and in the saturated flow region $100 \leq x \leq 800$. A height $h \approx 4$ in the integrals of (23) proved to be sufficient in order to recover an approximate uniform flow. Note that given the grid spacing $\Delta x=0.2$ and the number of 30 collocation points in $0 \leq y \leq h$, together with the sampling at each timestep, more than $2.10^{8}$ data had to be stored for each perturbation flow quantity.

In the case of the rigid wall, the wall pressure components $\hat{p}_{w}$ and $\partial \hat{p}_{w} / \partial y$ that appear in (23) are directly computed from the simulation using an identical sampling at the wall. For the elastic plates, on the contrary, these terms are modelled in order to take into account the compressible effects for the pressure induced by the plate motions. The different models that have been developed are detailed in section 4.1 .

A convenient quantity to evaluate the sound estimates has been com- 
puted, that is the radiated pressure spectral density

$$
P(Y, \omega)=\int|\hat{P}(k, Y, \omega)|^{2} d k .
$$

Note that in order to avoid singularities at the sonic wavenumber, as $\gamma$ appears in the denominator of formula (23), the wavenumber band $0.9 \leq$ $|k / M \omega| \leq 1.1$ has been excluded from the integration.

Considering the Mach number $M=0.012$ (corresponding to $U_{\infty}^{*}=$ $18 \mathrm{~m} / \mathrm{s}$ and water), for the lowest non zero wavenumber $\Delta k=2 \pi / L \approx 0.009$ the wavespeed $\omega / k$ is supersonic only for frequencies higher than 0.75 . Farfield radiation is hence negligible at this Mach number and near-field results are analyzed in the following, considering the value $Y=100$ in most of the computations.

\subsection{Acoustic wall pressure models}

In most hydrodynamic noise models involving vibrating walls based on wavenumber-frequency spectra, the flow pressure is computed as the sum of the pressure $p_{\text {rigid }}$ along a rigid wall in an equivalent configuration and a vibrating contribution $p_{a}$, that is

$$
\begin{array}{r}
\hat{p}(k, y, \omega)=\hat{p}_{\text {rigid }}(k, y, \omega)+\hat{p}_{a}(k, y, \omega) \\
\frac{\partial \hat{p}}{\partial y}(k, y, \omega)=\frac{\partial \hat{p}_{\text {rigid }}}{\partial y}(k, y, \omega)+\frac{\partial \hat{p}_{a}}{\partial y}(k, y, \omega)
\end{array}
$$

(cf. Graham [5] for a review). Inside the boundary layer, $\hat{p}_{a}$ is sought as being solution of the homogeneous wave equation

$$
\left(k^{2}-M^{2} \omega^{2}\right) \hat{p}_{a}-\frac{\partial^{2} \hat{p}_{a}}{\partial y^{2}}=0,
$$


which yields, using the $\gamma$ coefficient defined in (B.5),

$$
\hat{p}_{a}(k, y, \omega)=\frac{-i}{\gamma}\left[\frac{\partial \hat{p}_{a}}{\partial y}\right]_{y=0} e^{i \gamma y} .
$$

At the wall $y=0$ this component in the pressure decomposition becomes

$$
\hat{p}_{a}(k, 0, \omega)=\frac{-i}{\gamma}\left[\frac{\partial \hat{p}_{a}}{\partial y}\right]_{y=0} .
$$

Under the weak coupling assumption, which is generally used for self-noise prediction issues (see for instance Hariri \& Akylas [22], Graham [25] or Zheng [6]), the pressure normal derivative at the wall is estimated by writing merely

$$
\left[\frac{\partial p_{a}}{\partial y}\right]_{y=0}=-\left[\frac{\partial v}{\partial t}\right]_{y=0},
$$

which gives in the spectral domain

$$
\left[\frac{\partial \hat{p}_{a}}{\partial y}\right]_{y=0}=\omega^{2} \hat{\eta}
$$

by taking into account the kinematic condition (8). Hence, the vibrating component at the wall $(y=0)$ is written when using the weak coupling assumption as

$$
\hat{p}_{a}(k, 0, \omega)=\frac{-i \omega^{2}}{\gamma} \hat{\eta} .
$$

However, when nonlinearities result from the coupling between the flow and the plate, the simple relation (34) may not be reliable anymore. Our solution procedure provides a wall pressure gradient $\partial \hat{p}_{w} / \partial y$ associated with the full coupling between the boundary layer and the plate. Consequently, when taking into account full coupling, the relation (31) is written at the wall and the resulting expression for the gradient $\partial \hat{p}_{a} / \partial y$ is used in (33). This gives rise to the expression

$$
\hat{p}_{a}(k, 0, \omega)=-\frac{i}{\gamma}\left[\frac{\partial \hat{p}_{w}}{\partial y}-\frac{\partial \hat{p}_{w, r i g i d}}{\partial y}\right],
$$


rather than (36), with $\partial \hat{p}_{w, \text { rigid }} / \partial y$ the pressure gradient for the flow along the rigid wall. The wavenumber-frequency spectra for both $\omega^{2} \hat{\eta}$ and $\frac{\partial \hat{p}_{w}}{\partial y}-$ $\frac{\partial \hat{p}_{w, \text { rigid }}}{\partial y}$ are shown in figure 16. Both spectra have similar modal peaks but when strong coupling is considered the convective part is much more pronounced.

The pressure decomposition (30), (31) has been considered in (23) to compute the acoustic pressure $\hat{P}(k, Y, \omega)$. When the weak coupling hypothesis is made, $\hat{p}_{a}$ and $\partial \hat{p}_{a} / \partial y$ are given respectively by (35), (36), and when strong coupling is considered the acoustic pressure (37) is used. In order to highlight the effect of the wall pressure, the Fourier transform of the Lighthill's tensor for the rigid wall has been taken into account for both coupling assumptions.

The radiated pressure spectral densities (at $Y=100$ ) are depicted in figure 17 for plate 1 and compared with the case of the rigid wall. For the latter, the highest pressure levels are reached at the forcing frequencies and at their harmonics. Similar peaks are visible in the case of the compliant wall, but the pressure densities are now clearly dominated by a large peak at its natural frequency 0.26. Also, the acoustic levels are globally much higher in the mid- and high-frequency ranges than in the rigid case. Comparing the spectral densities for plate 1, the levels are seen to depend on the coupling model, although the modal peaks at $\omega=0.26$ are identical. In the low frequency range, the full coupling assumption gives rise to higher acoustic pressure levels, both at the forcing frequencies and the intermediate ones, than the weak coupling model. In the high frequency range, the acoustic levels seem to be slightly overpredicted under the weak coupling assumption.

Figures 18 (a), (b), (c) show isolines of the radiated pressure in the phys- 

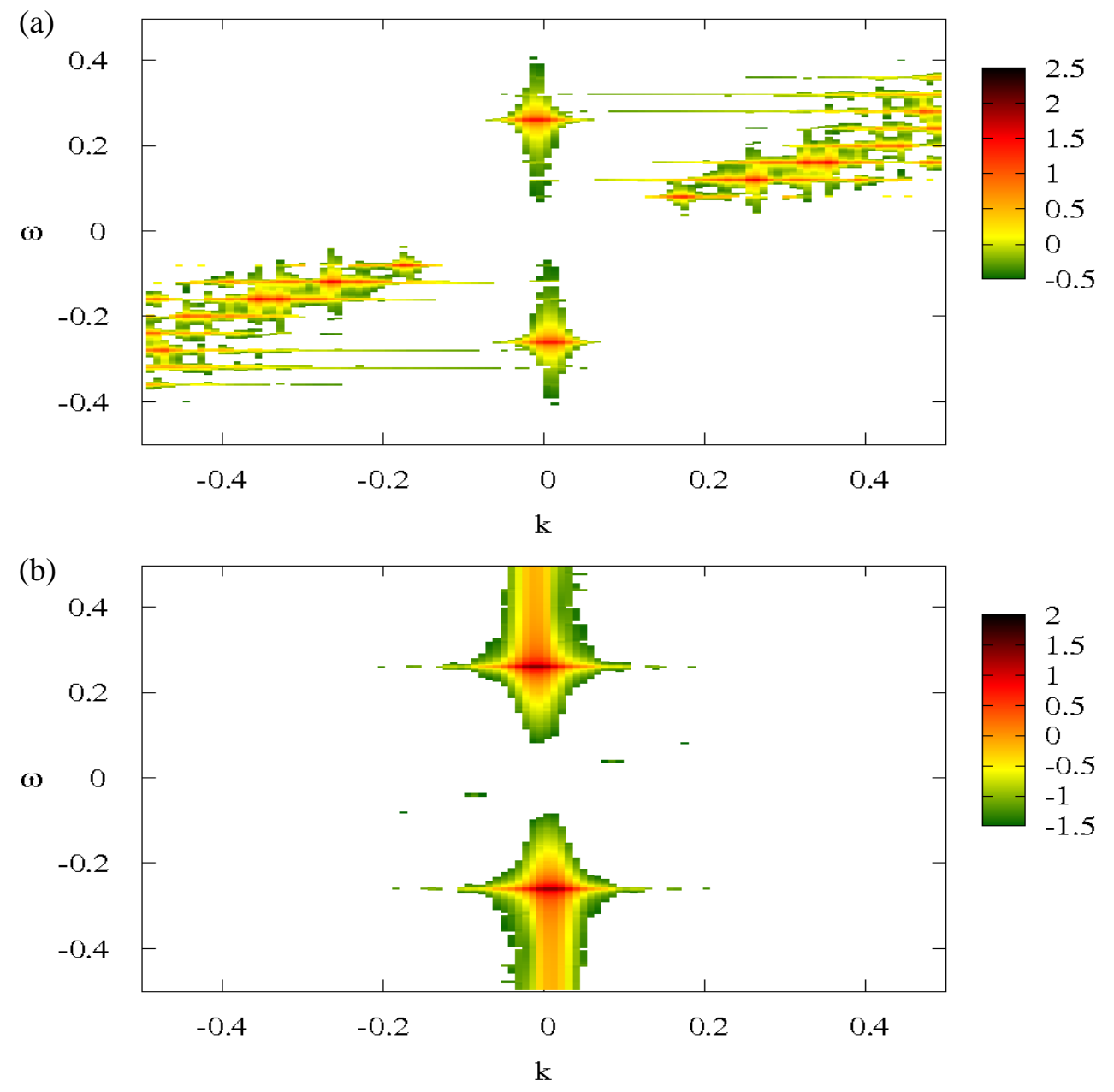

Figure 16: $\log \left|\frac{\partial \hat{p}_{a}}{\partial y}\right|$ for plate 1 at $y=0$ (a) $\frac{\partial \hat{p}_{a}}{\partial y}=\frac{\partial \hat{p}_{w}}{\partial y}-\frac{\partial \hat{p}_{w} \text {, rigid }}{\partial y}$, color scale ranging from -0.5 to 2.5 (b) $\frac{\partial \hat{p}_{a}}{\partial y}=\omega^{2} \hat{\eta}$, color scale ranging from -1.5 to 2 . 


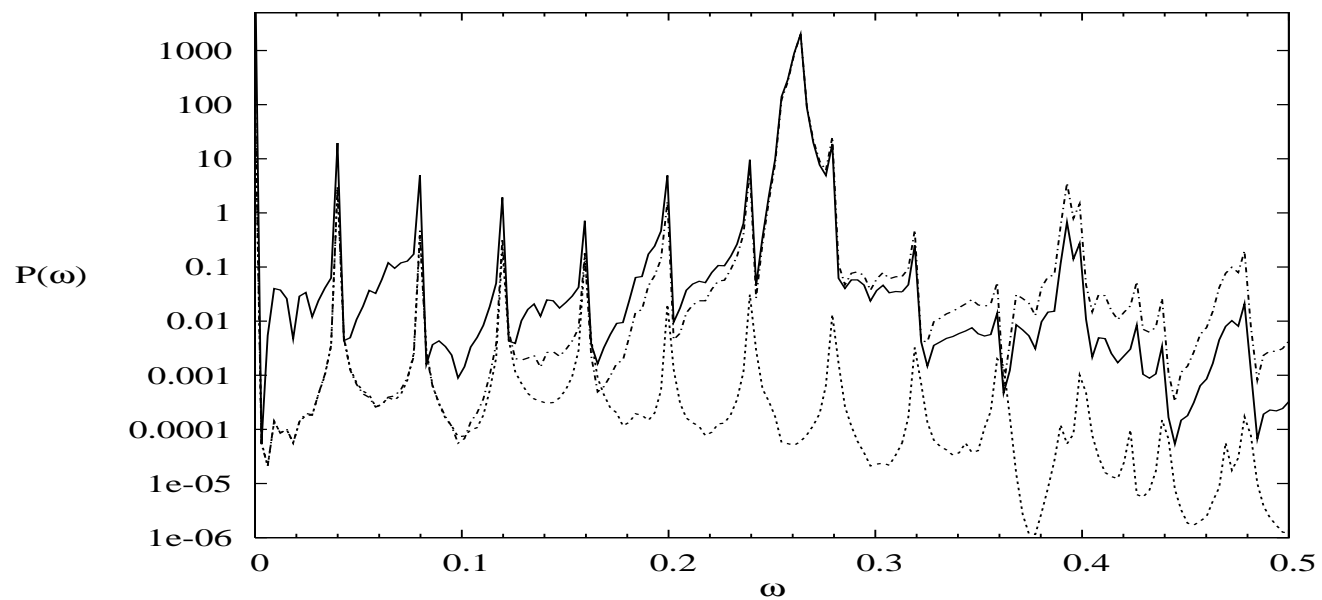

Figure 17: Radiated pressure spectral densities at $Y=100$ for plate 1 under the full coupling (-) and weak coupling $(-\cdot-)$ assumptions, and for the rigid wall $(\cdots)$.

ical space, for the flow over the rigid wall as well as for the weak and strong coupling models. The frequency $\omega=0.142$ corresponding to an intermediate value between two forcing frequency peaks has been chosen (cf. figure 17). For the rigid wall there is hardly any sound at some distance from the wall. The sound levels are clearly enhanced by the full coupling, exhibiting non negligible levels at $Y=200$ and beyond.

The same general trend arises from the comparison between the weak and strong coupling assumptions in the case of plate 2, although the differences are much less pronounced than for plate 1 . Both radiated pressure spectral densities, shown in figure 19, exhibit modal peaks of moderate amplitude at the natural frequencies 0.16 (which is also a forcing frequency) and 0.42 . The acoustic levels at intermediate frequencies are increased as well, compared to the case of the rigid wall. 

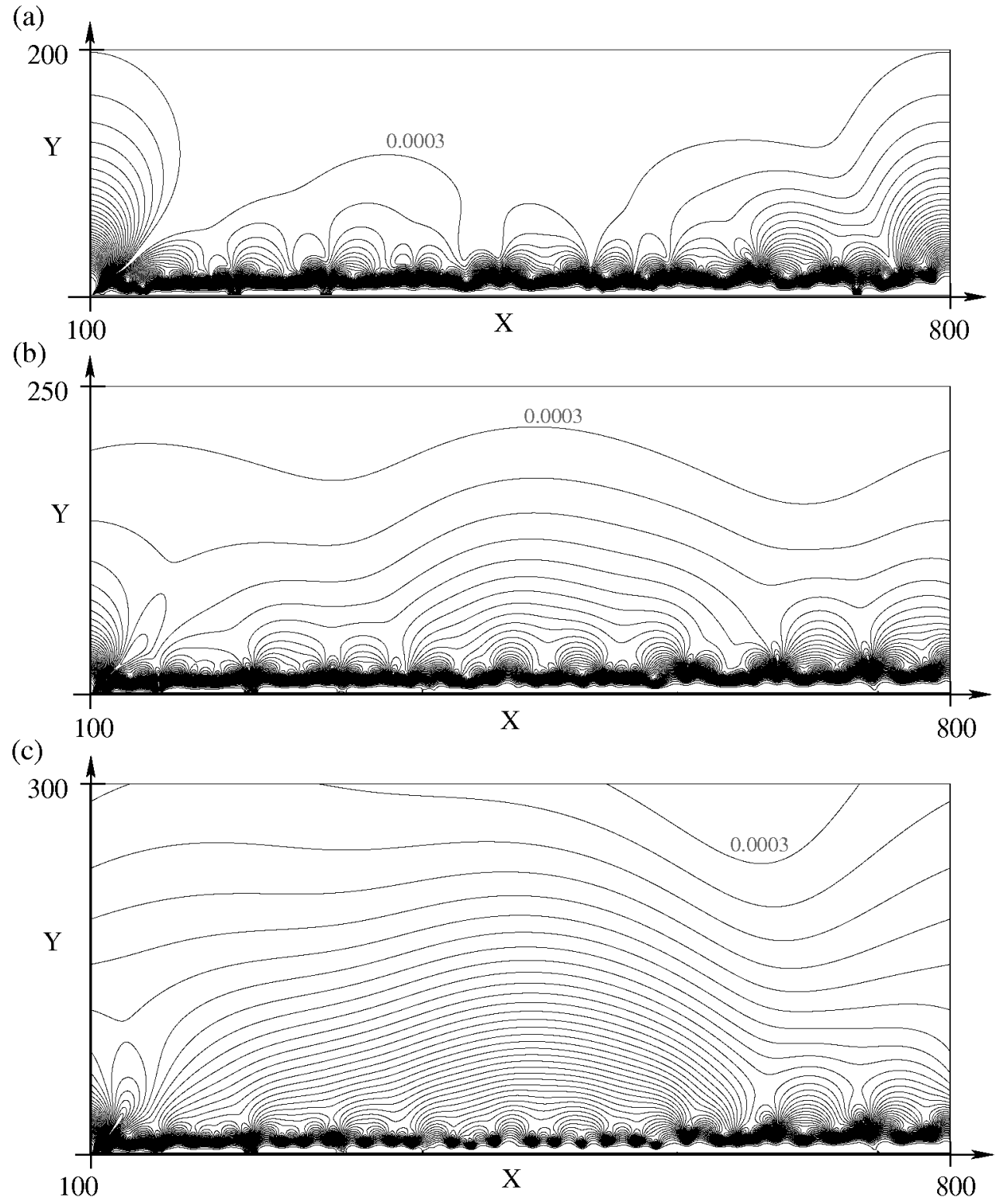

Figure 18: Isolines of the radiated pressure levels at $\omega=0.142$, ranging from 0.0003 to 0.03: (a) rigid wall, (b) plate 1 under the weak coupling assumption, (c) plate 1 under the full coupling assumption. 


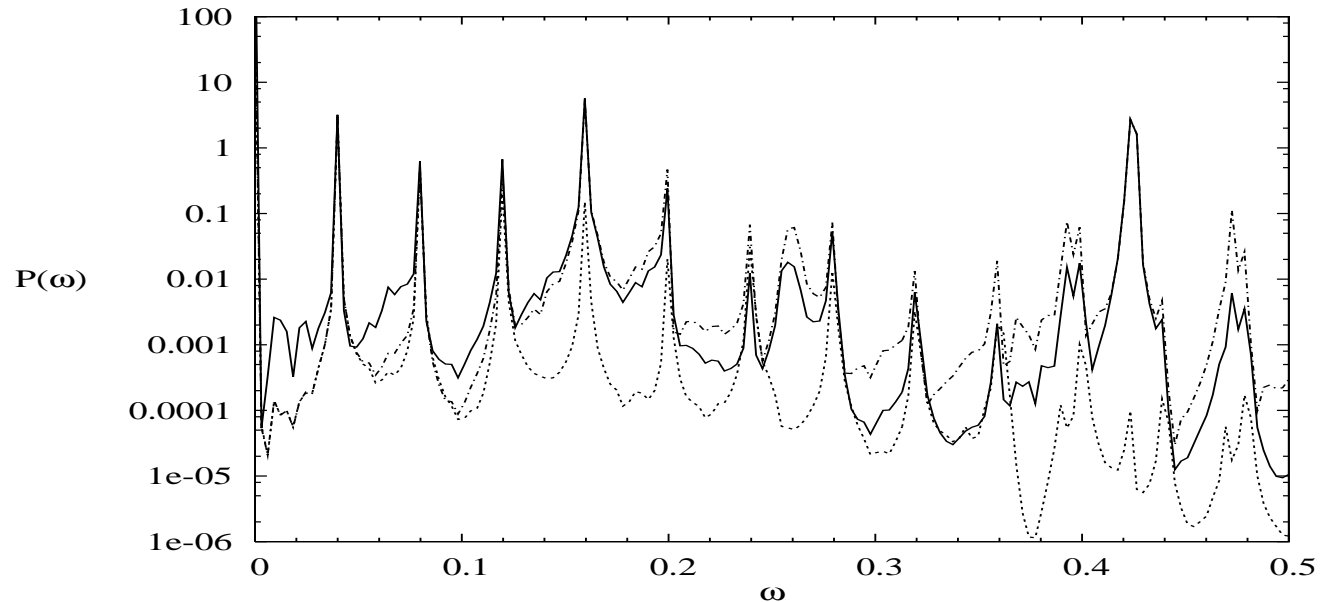

Figure 19: Radiated pressure spectral densities for plate 2 at $Y=100$ under the full coupling (-) and weak coupling $(-\cdot-)$ assumptions, and for the rigid wall $(\cdots)$.

\subsection{Wall shear stress contribution}

As mentioned in section 2.2, the above radiated pressure levels have been obtained neglecting the contribution due to the wall shear stress fluctuations. Equation (24) provides the Fourier-transformed radiated pressure term $\hat{P}_{v}(k, Y, \omega)$ associated with the wall shear stress. The magnitude of this term compared with the radiated pressure computed in the previous section has been assessed for plate 1, for the full coupling model only. The result is shown in figure 20, which compares the radiated spectral densities of $\hat{P}$ and $\hat{P}_{v}$ at $Y=100$. In the whole frequency range, except at a few very low frequency values, the levels obtained for $\hat{P}_{v}$ are seen to be several orders of magnitude smaller than the levels for $\hat{P}$, and may hence be neglected for sound radiation estimates. Indeed, the spectral density for $\hat{P}+\hat{P}_{v}$ would superimpose to the curve with $\hat{P}$ and it is not depicted in the figure. 


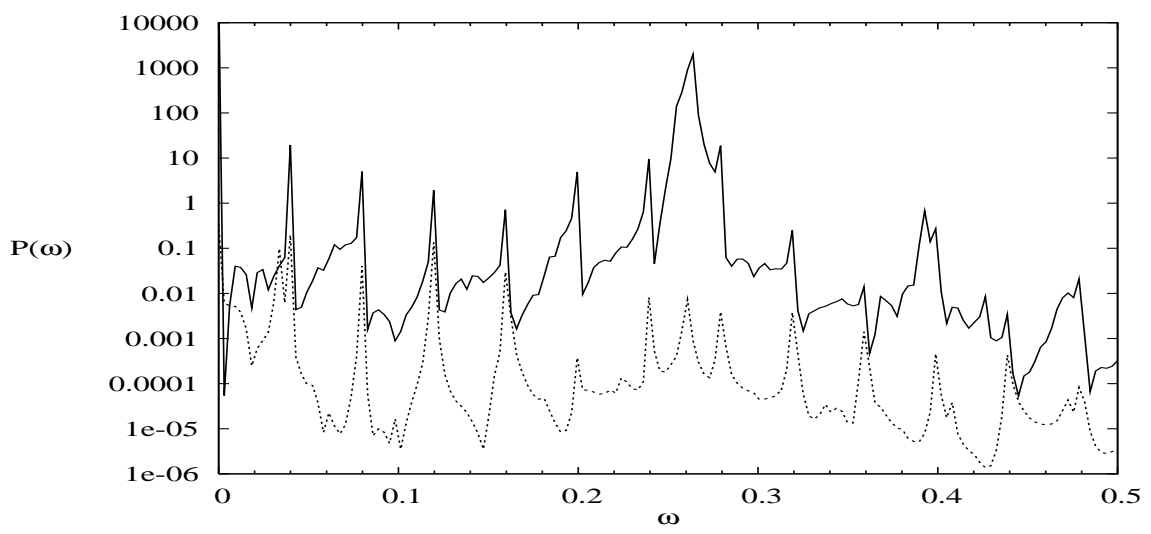

Figure 20: $\hat{P}(-)$ and $\hat{P}_{v}(\cdots)$ spectral densities for plate 1 under the full coupling assumption at $Y=100$.

\subsection{Lighthill's tensor from the coupled simulation}

In order to highlight the effect of the coupling assumption involving the wall pressure, the previous results have been obtained using the Lighthill's tensor $\mathcal{T}_{i j}$ for the simulation with the rigid wall, as for instance in [6]. Performing coupled simulations involving elastic plates, the velocity fluctuations $\left(u^{\prime}, v^{\prime}\right)$ resulting from the fluid-structure interaction may be directly used in (11) and (12) to compute a modified Lighthill's tensor. This so-called 'elastic' Lighthill's tensor (in contrast with the former 'rigid' tensor) has been considered for the case of plate 1, under the full coupling assumption. The corresponding acoustic field has been computed and is compared in figure 21 with the result of section 4.1. Both spectral densities exhibit similar acoustic levels at the forcing frequencies and their harmonics. To take into account the velocity fluctuations resulting from the full coupling between the flow and the plate motions in the construction of the Lighthill's tensor is however seen to enlarge the modal peak and to give rise to higher acoustic levels at 


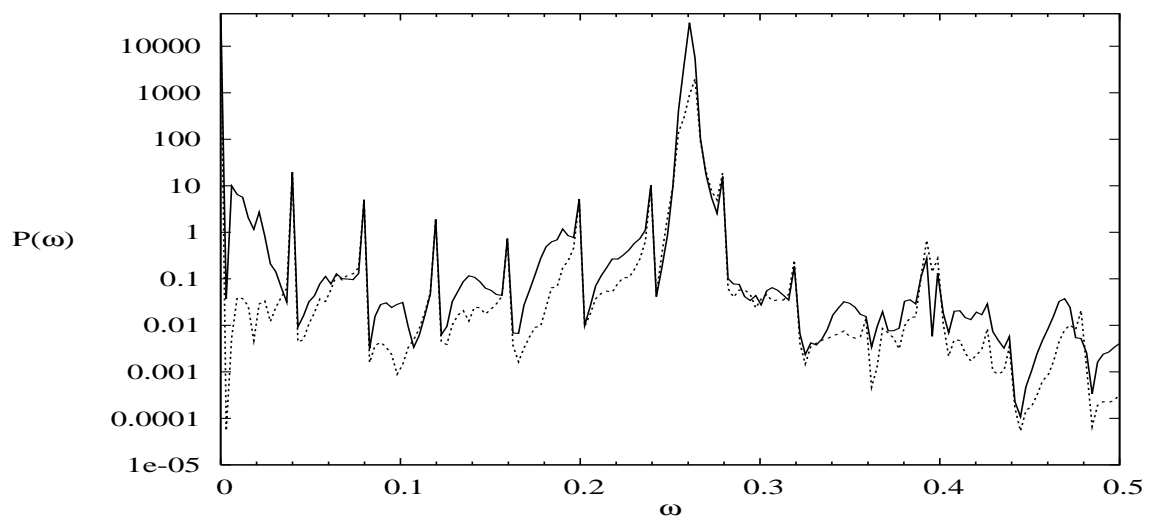

Figure 21: Radiated pressure spectral densities for plate 1 under the full coupling assumption at $Y=100 \mathrm{using}$ the elastic Lighthill's tensor $(-)$ and the rigid tensor $(\cdots)$.

the intermediate frequencies in the whole frequency range. $t$ These effects are highlighted when comparing the corresponding spatial distributions of radiated pressures $\hat{P}(X, Y, \omega)$ at the same distance $Y=100$ for given intermediate frequencies. The cases of a very low frequency $\omega=0.02$ and of the same frequency $\omega=0.142$ as considered in figure 18 are depicted in figures 22 (a) and (b) respectively. At both frequencies the levels induced by the elastic Lighthill's tensor are seen to dominate throughout the spatial domain.

\section{Conclusion}

The prediction of self noise induced by the interaction of a boundarylayer flow with a flexible wall is in general based on a weak coupling assumption. The models commonly available consider a linear superimposition of the wavenumber-frequency spectrum for the (turbulent) boundary layer over a rigid wall, and an acoustic pressure fluctuation due to the plate motion, 

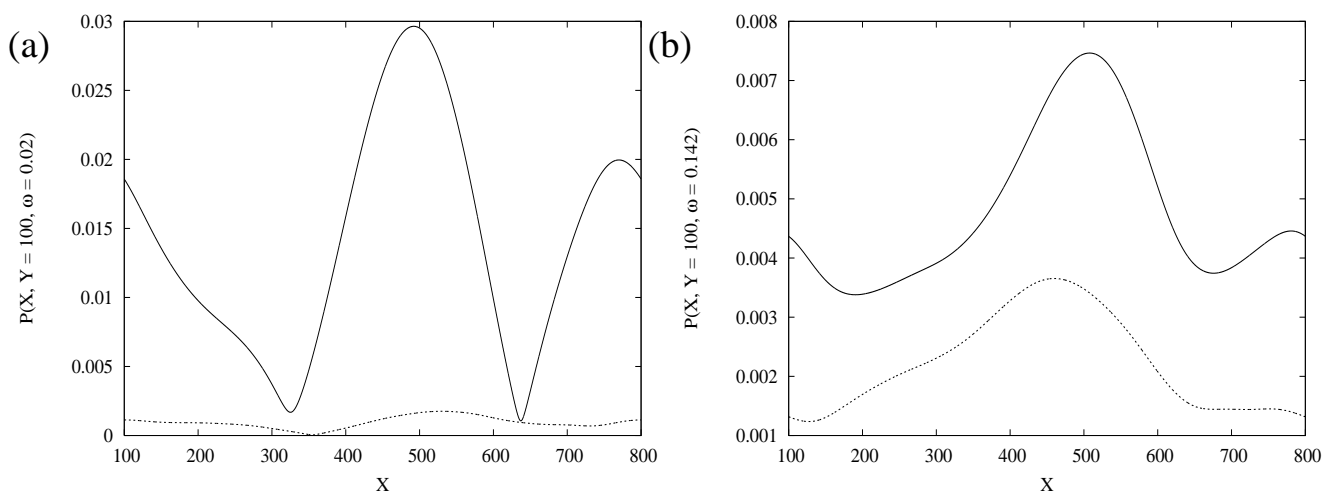

Figure 22: Spatial distributions of the radiated pressure $\hat{P}(X, Y, \omega)$ at $Y=100$ for plate 1 under the full coupling assumption using the elastic Lighthill's tensor $(-)$ and the rigid tensor ( ...) (a) $\omega=0.02$ (b) $\omega=0.142$.

by making the assumption that the wall pressure normal gradient is only linearly coupled to the wall-normal velocity.

To perform a simulation of a turbulent boundary layer interacting with a flexible plate without simplifying assumptions would hardly be feasible, or would at least demand considerable numerical efforts. The present analysis, addressing the noise radiation issue for the model of a two-dimensional nonlinearly saturated boundary-layer along an elastic plate, may hence be considered as a first attempt to assess the reliability of different coupling assumptions. As in [6], the flexible wall effects have been represented by the Green function, used to solve the Lighthill's equation, for data from the numerical simulation of the fully coupled fluid-structure system. It appears that for nonlinearly saturated flow states, nonlinear terms arise through the coupling between the boundary-layer flow and the plate motion, affecting in turn the wavenumber-frequency spectrum of the pressure gradient at the wall. The acoustic pressure fluctuation due to the wall motion is modified 
accordingly, the effect on the radiated sound depends, however, on the plate characteristics. For the low Mach number considered, owing to the hydrodynamic context of the analysis, the radiated pressure levels at moderate distances from the wall have been computed. For the different coupling hypotheses, our results are in agreement with the general trend that the sound levels are enhanced in the presence of a flexible wall, reported for instance in [6] under the weak coupling assumption and considering experimental data for the turbulent wall-pressure spectra.

Addressing the strong versus weak coupling models, for a plate with high stiffness and one dominant natural frequency (plate 1 in the above sections), we found that the radiated sound levels are enhanced in the low-frequency range when the full coupling is taken into account, in particular at intermediate frequencies with respect to those of the harmonic forcing of the boundary-layer. In the high-frequency range however the radiated pressure levels resulting from the weak coupling hypothesis slightly dominate. When considering an elastic plate without stiffness and with a natural frequency within the range of the harmonic flow forcing (plate 2), the weak and strong coupling results are almost identical at low-frequencies. At higher frequencies the radiated pressure levels are again slightly overpredicted when the weak coupling assumption is made. The contribution to the radiated pressure due to wall shear stress fluctuations appears to be negligible for the flow over the compliant walls considered in the present analysis.

In radiated pressure models for compliant walls, rigid-wall turbulent fluctuating velocity quantities are considered as sources in the Lighthill's equation. For the academic configuration considered here, these quantities can 
be computed as arising from the coupling between the flow perturbation and the wall motion. Combining this approach with the previous full coupling assumption for the fluctuating wall pressure quantities, we obtain an acoustic model which takes even more into account the fluid-structure coupling. The resulting radiated pressure levels are shown to be enhanced in comparison to the previous results regarding elastic plates. It would be hazardous to directly interpret the present findings with regard to real flow situation, exhibiting three-dimensionality and turbulence. However, the results indicate that wavenumber-frequency models for radiated sound are likely to depend on the specific coupling assumption used to take into account the wall motion.

\section{Acknowledgements}

The authors gratefully acknowledge Thales Underwater Systems and DCNS for their financial support to this work.

\section{Appendix A. Coupling between pressure and wall displacement}

Once the spatial operators in (1) and (2) are discretized and applying the second-order backward difference formula for the time derivative, the velocity field $\mathbf{u}^{n+1}$ and the pressure $p^{n+1}$ at the new time $(n+1) \Delta t$ are solutions of

$$
\begin{aligned}
\left(\frac{1}{R e} \bar{\nabla}^{2}-\frac{3}{2 \Delta t}\right) \mathbf{u}^{n+1} & =\bar{\nabla} p^{n+1}+[\mathbf{f}]^{n, n-1} \\
\bar{\nabla} \cdot \mathbf{u}^{n+1} & =-\left[\mathbf{G}_{\eta} \cdot \mathbf{u}\right]^{n, n-1}
\end{aligned}
$$

where []$^{n, n-1}$ means that the terms inside the brackets, containing the nonlinear as well as the metric terms, are evaluated according to the explicit second-order Adams-Bashforth rule and the system has to be solved for the 
time-dependent wall displacement $\eta$. Applying second-order finite-differences to $\partial^{4} / \partial x^{4}$ in (6), together with second order finite-differences formulae for the first and second-order derivatives in time, the system to be solved for $\eta^{n+1}$ may be written formally as

$$
L \eta^{n+1}=\eta^{n, n-1}+\sigma_{p}, \quad \sigma_{p}=-p^{n+1}+\tau^{n, n-1}
$$

with $\tau^{n, n-1}$ the Adams-Bashforth rule applied to the viscous normal stress. Knowing the flow quantities and the wall displacement up to time step $n$, a first intermediate pressure $p^{*}$ at time step $n+1$ is obtained solving the discretized version of the Poisson equation (9) for the pressure

$$
\bar{\nabla}^{2} p^{*}=\left[-L_{\eta} p+2\left[\frac{\partial u}{\partial \bar{x}} \frac{\partial v}{\partial \bar{y}}-\frac{\partial u}{\partial \bar{y}} \frac{\partial v}{\partial \bar{x}}\right]\right]^{n, n-1} .
$$

The Neumann boundary condition for $p^{*}$ is given by projection of the momentum equations normal to the wall. The estimated pressure $p^{*}$ provides $\mathbf{u}^{*}$ as well as $\eta^{*}$. In order to recover a divergence-free velocity field at the new time step $n+1$, the correction of the pressure $\phi$ is such that

$$
\nabla \phi=-\frac{3}{2 \Delta t}\left(\mathbf{u}^{n+1}-\mathbf{u}^{*}\right)
$$

Taking the divergence of the equation one gets in physical coordinates

$$
\nabla^{2} \phi=\frac{3}{2 \Delta t} \nabla \cdot \mathbf{u}^{*}
$$

and applying the mapping one has to solve

$$
\left(\bar{\nabla}^{2}+L_{\eta}\right) \phi=\frac{3}{2 \Delta t}\left(\nabla \cdot \mathbf{u}^{*}+\mathbf{G}_{\eta} \cdot \mathbf{u}^{*}\right)
$$

where the operators $L_{\eta}$ and $\mathbf{G}_{\eta}$ are defined in (5). The equation above is solved iteratively with

$$
\bar{\nabla}^{2} \phi^{(k+1)}=\frac{3}{2 \Delta t}\left(\nabla \cdot \mathbf{u}^{*}+\mathbf{G}_{\eta^{(k)}} \cdot \mathbf{u}^{*}\right)-L_{\eta^{(k)}}, \quad k=1, \cdots, K
$$


with $\eta^{(k)}$ solution of the plate equation with the right-hand side

$$
\sigma_{p}=-\left(p^{*}+\phi^{(k)}\right)+\tau^{n, n-1}
$$

In general $K=3$ iterations proved to be sufficient to achieve an error of order $\Delta t^{2}$. The system (A.1)-(A.2) is hence solved up to the overall truncation error in time and the wall displacement as well as the divergence-free velocity field at the new time step $n+1$ are

$$
\begin{aligned}
p^{n+1} & =p^{*}+\phi^{(K)} \\
\mathbf{u}^{n+1} & =\mathbf{u}^{*}-\frac{2 \Delta t}{3}\left(\bar{\nabla} \phi^{(K)}+\mathbf{G}_{\eta^{(K)}} \phi^{(K)}\right) \\
\eta^{n+1} & =\eta^{(K)} .
\end{aligned}
$$

The equation (A.3) is solved using a homogeneous Neumann boundary condition and hence the boundary-integral zero mass-flux condition

$$
\int_{\delta \Omega} \mathbf{u}^{*} d s=0
$$

has to be satisfied. This could not be achieved by solely considering the advection condition at outflow

$$
\frac{\partial \mathbf{u}}{\partial t}+U_{c} \frac{\partial \mathbf{u}}{\partial x}=0
$$

and the kinematic condition $v=\partial \eta / \partial t$ has been mirrored at the upper boundary $y=y_{\max }$. This condition leads to a (small) oscillation of $v$ in the upper region which does not affect the fluid-structure interaction inside the boundary layer. Indeed, as the flow is uniform in the upper part of the computational domain, the gradient $\partial v / \partial y$ vanishes in the region adjacent to $y_{\max }$. 


\section{Appendix B. Green function calculation}

According to equation (15) the Fourier transform (20) of the Green function is easily seen to be solution of

$$
\left(k^{2}-M^{2} \omega^{2}\right) \hat{G}-\frac{\partial^{2} \hat{G}}{\partial y^{2}}=e^{-i(k X-\omega T)} \delta(Y-y) .
$$

Using the product theorem of Fourier transforms, the boundary condition (17) can equivalently be written

$$
\int\left(\frac{\partial \hat{G}}{\partial y} \hat{p}_{w}(-k,-\omega)-\hat{G} \frac{\partial \hat{p}_{w}}{\partial y}(-k,-\omega)\right) d k d \omega=0
$$

with $\hat{p}_{w}(k, \omega), \frac{\partial \hat{p}_{w}}{\partial y}(k, \omega)$ the Fourier-transform of the wall fluctuation pressure and its wall-normal derivative, respectively. According to (B.1), the function $\hat{g}=\hat{G} / e^{-i(k X-\omega T)}$ is solution of

$$
\left(M^{2} \omega^{2}-k^{2}\right) \hat{g}+\frac{\partial^{2} \hat{g}}{\partial y^{2}}=-\delta(Y-y)
$$

and the condition (B.2) is satisfied if

$$
\frac{\partial \hat{g}}{\partial y}(k, 0, \omega) \hat{p}_{w}(-k,-\omega)-\hat{g}(k, 0, \omega) \frac{\partial \hat{p}_{w}}{\partial y}(-k,-\omega)=0 .
$$

To solve the equation for $\hat{g}$, a root of $\gamma^{2}=M^{2} \omega^{2}-k^{2}$ has to be chosen and we define

$$
\gamma= \begin{cases}\omega \sqrt{M^{2}-\frac{k^{2}}{\omega^{2}}} & \text { if } M^{2}-\frac{k^{2}}{\omega^{2}} \geq 0, \\ i \sqrt{k^{2}-M^{2} \omega^{2}} & \text { if } M^{2}-\frac{k^{2}}{\omega^{2}}<0 .\end{cases}
$$

Hence, when $\gamma$ is real, that is for supersonic conditions $|\omega / k| \geq 1 / M, \gamma$ has the sign of $\omega$ and if $\gamma$ is imaginary the root is chosen such that $e^{i \gamma y}$ vanishes 
at infinity (cf. Dowling [10], Tam [11]). By prescribing a decaying wave behaviour at infinity, the solution $\hat{g}$ is (for $Y>0$ )

$$
\begin{array}{lr}
\hat{g}_{+}=A_{+} e^{i \gamma y}, & y>Y \\
\hat{g}_{-}=A_{-} e^{i \gamma y}+B_{-} e^{-i \gamma y}, & 0 \leq y<Y
\end{array}
$$

The coefficients $A_{+}, A_{-}, B_{-}$are such that continuity $\hat{g}_{+}=\hat{g}_{-}$as well as the jump condition $\partial \hat{g}_{+} / \partial y-\partial \hat{g}_{-} / \partial y=-1$ are satisfied at $y=Y$. The boundary condition (B.4) for $\hat{g}_{-}$at $y=0$ provides the third equation and the coefficients can hence be determined. One recovers the Fourier-transformed Green function expression

$$
\hat{G}=-e^{i(\omega T-K X+\gamma Y)}\left(\frac{\left(i \gamma \hat{p}_{w}^{*}+\frac{\partial \hat{p}_{w}^{*}}{\partial y}\right) e^{i \gamma y}}{2 i \gamma\left(i \gamma \hat{p}_{w}^{*}-\frac{\partial \hat{p}_{w}^{*}}{\partial y}\right)}+\frac{e^{-i \gamma y}}{2 i \gamma}\right), 0 \leq y<Y
$$

and

$$
\hat{G}=-e^{i(\omega T-K X+\gamma y)}\left(\frac{\left(i \gamma \hat{p}_{w}^{*}+\frac{\partial \hat{p}_{w}^{*}}{\partial y}\right) e^{i \gamma Y}}{2 i \gamma\left(i \gamma \hat{p}_{w}^{*}-\frac{\partial \hat{p}_{w}^{*}}{\partial y}\right)}+\frac{e^{-i \gamma Y}}{2 i \gamma}\right), \quad y>Y .
$$

(in the above equations the superscript $*$ denotes the complex conjugate, that is $\hat{p}_{w}^{*}=\hat{p}_{w}(-k,-\omega)$ etc. $)$. For $y>Y$ and considering the limit case $Y=0$, one recovers the expression provided for instance in [6].

\section{References}

[1] G.M. Corcos, The structure of the turbulent pressure field in boundarylayer flow, J. Fluid Mech. 18 (1964) 353-378.

[2] D.M. Chase, Modeling the wavevector-frequency spectrum of turbulent boundary layer wall pressure, J. Sound Vib. 70 (1980) 29-67. 
[3] D.M. Chase, The character of the turbulent wall pressure at subconvective wavenumbers and a suggested comprehensive model, J. Sound Vib. 112 (1987) 125-147.

[4] J.E. Ffowcs Williams, Boundary-layer pressures and the Corcos model: a development to incorporate low wavenumber constraints, J. Fluid Mech. $125(1982)$ 9-25.

[5] W.R. Graham, A comparison of models for the wavenumber-frequency spectrum of turbulent boundary layer pressures, J. Sound Vib. 206(4) (1997) 541-565.

[6] Z.C. Zheng, Effects of flexible walls on radiated sound from a turbulent boundary layer, J. Fluids Struct. 18 (2003) 93-101.

[7] Z.C. Zheng, Effects of compliant coatings on radiated sound from a rigidwall turbulent boundary layer, J. Fluids Struct. 19 (2004) 933-941.

[8] M.J. Lighthill, On sound generated aerodynamically. I: General theory, Proc. Roy. Soc. Lond. A 211 (1952) 564-587.

[9] M.J. Lighthill, On sound generated aerodynamically. II: Turbulence as a source of sound, Proc. Roy. Soc. Lond. A 221 (1954) 1-32.

[10] A.P. Dowling, Flow-acoustic interaction near a flexible wall, J. Fluid Mech. 128 (1983) 181-198.

[11] C.K.W. Tam, Intensity, spectrum and directivity of turbulent boundary layer noise, J. Acoust. Soc. Am. 57 (1975) 25-34. 
[12] P.W Carpenter, A.D. Garrad, The hydrodynamic stability of flow over Kramer-type compliant surfaces. Part 1. Tollmien-Schlichting instabilities, J. Fluid Mech. 155 (1985) 465-510.

[13] C. Davies, P.W. Carpenter, Numerical simulation of the evolution of Tollmien-Schlichting waves over finite compliant panels, J. Fluid Mech. 335 (1997) 361-392.

[14] M. Gad-el Hak, Compliant coatings: the simpler alternative, Exp. Therm. Fluid Sci. 16 (1998) 141-156.

[15] O. Wiplier, U. Ehrenstein, Numerical simulation of linear and nonlinear disturbance evolution in a boundary layer with compliant walls, J. Fluids Struct. 14 (2000) 157-182.

[16] M. Wang, S.K. Lele, P. Moin, Sound radiation during local laminar breakdown in a low-Mach-number boundary layer, J. Fluid Mech. 319 (1996) 197-218.

[17] S. Xu, D. Rempfer, J. Lumley, Turbulence over a compliant surface: numerical simulation and analysis, J. Fluid Mech. 478 (2003) 11-34.

[18] Y.P. Guo, Application of the Ffowcs Williams/Hawkings equation to two-dimensional problems, J. Fluid Mech. 403 (2000) 201-221.

[19] M. Marquillie, U. Ehrenstein, Numerical simulation of separating boundary-layer flow, Comput. Fluids 31 (2002) 683-693.

[20] M. Marquillie, \& U. Ehrenstein, On the onset of nonlinear oscillations in a separating boundary-layer flow, J. Fluid Mech. 490 (2003) 169-188. 
[21] J. Kim, P. Moin, Application of a fractional-step method to incompressible Navier-Stokes equations, J. Comput. Phys. 59 (1985) 308-323.

[22] H.H. Hariri, T.R. Akylas, Mean-flow effects on the low-wavenumber wall-pressure spectrum of a turbulent boundary-layer over a compliant surface, J. Acoust. Soc. Am. 77(5) (1985) 1840-1844.

[23] K. Shariff, M. Wang, A numerical experiment to determine whether surface shear-stress fluctuations are a true sound source, Phys. Fluids 17 (2005) 107105.

[24] Z. Hu, C.L. Morfey, N.D. Sandham, Sound radiation from a turbulent boundary layer, Phys. Fluids 18 (2006) 098101.

[25] W.R. Graham, Boundary layer induced noise in aircraft, part I: the flat plate model, J. Sound Vib. 192(1) (1996) 101-120. 